ARTICLE

https://doi.org/10.1038/s41467-018-08276-6

\title{
A selective inhibitor of mitofusin 1- $\beta$ IIPKC association improves heart failure outcome in rats
}

Julio C.B. Ferreira (1) 1,2, Juliane C. Campos ${ }^{1}$, Nir Qvit ${ }^{2}$, Xin Qi (1) ${ }^{2,3}$, Luiz H.M. Bozi1, Luiz R.G. Bechara1, Vanessa M. Lima', Bruno B. Queliconi (1) ${ }^{4}$, Marie-Helene Disatnik², Paulo M.M. Dourado5, Alicia J. Kowaltowski ${ }^{4} \&$ Daria Mochly-Rosen (1) ${ }^{2}$

We previously demonstrated that beta II protein kinase $C$ ( $\beta$ IIPKC) activity is elevated in failing hearts and contributes to this pathology. Here we report that $\beta$ IIPKC accumulates on the mitochondrial outer membrane and phosphorylates mitofusin 1 (Mfn1) at serine 86. Mfn1 phosphorylation results in partial loss of its GTPase activity and in a buildup of fragmented and dysfunctional mitochondria in heart failure. $\beta$ IIPKC siRNA or a $\beta$ IIPKC inhibitor mitigates mitochondrial fragmentation and cell death. We confirm that Mfn1- $\beta$ IIPKC interaction alone is critical in inhibiting mitochondrial function and cardiac myocyte viability using SAM $\beta A$, a rationally-designed peptide that selectively antagonizes Mfn1- $\beta$ IIPKC association. SAM $\beta A$ treatment protects cultured neonatal and adult cardiac myocytes, but not Mfn1 knockout cells, from stress-induced death. Importantly, SAM $\beta$ A treatment re-establishes mitochondrial morphology and function and improves cardiac contractility in rats with heart failure, suggesting that SAM $\beta A$ may be a potential treatment for patients with heart failure.

\footnotetext{
${ }^{1}$ Department of Anatomy, Institute of Biomedical Sciences, University of Sao Paulo, Sao Paulo 05508-000 SP, Brazil. ${ }^{2}$ Department of Chemical and Systems Biology, Stanford University School of Medicine, Stanford 94305-5174 CA, USA. ${ }^{3}$ Department of Physiology \& Biophysics, Case Western Reserve University, Cleveland 44106 OH, USA. ${ }^{4}$ Departamento de Bioquímica, Instituto de Química, Universidade de Sao Paulo, Sao Paulo 05508-000 SP, Brazil. ${ }^{5}$ Heart Institute, University of Sao Paulo, Sao Paulo 05403-010 SP, Brazil. Correspondence and requests for materials should be addressed to J.C.B.F. (email: jcesarbf@usp.br) or to D.M.-R. (email: mochly@stanford.edu)
} 
D espite advances in clinical and pharmacological interventions, acute myocardial infarction with subsequent left ventricular dysfunction and heart failure continues to be a major cause of morbidity and mortality worldwide ${ }^{1,2}$. Therefore, the identification of novel therapeutic targets that improve cardiac function in patients with myocardial infarction-induced heart failure remains a major priority.

Protein kinases are key players in cellular signaling ${ }^{3}$. They work as intracellular nodes where signals converge to and serve as multi-effector triggers and/or brakes. Through phosphorylation of specific substrates, protein kinases can regulate a wide array of intracellular pathways that control cardiac metabolism, contractility, remodeling, and survival. Therefore, they are attractive molecular targets for cardiovascular diseases. However, the vast majority of protein kinase inhibitors targets its ATP binding pocket, a highly conserved region across the kinome, and often induces cardiotoxicity by inhibiting unintended kinases ${ }^{4,5}$. Moreover, regardless of its upstream signaling, the same protein kinase can activate multiple signaling pathways simultaneously (essential and detrimental) during disease progression and therefore affect the effectiveness and safety of PKC inhibitors in the long-term. The screen or design of molecules that competitively disrupt a specific protein-protein interaction (kinase-substrate) has been considered an important approach to develop more feasible drugs that selectively affect only detrimental kinasesubstrate interactions in cardiac pathophysiology $y^{5-7}$.

Protein kinase C (PKC) is a family of closely related serinethreonine protein kinases involved in a variety of acute and chronic cardiovascular diseases (i.e., ischemia-reperfusion injury, hypertension, and heart failure $)^{8}$. We have previously demonstrated that treatment of isolated hearts, rodents, or humans with rational design peptides that inhibit protein-protein interaction between a specific PKC and its anchor protein protects the heart against acute ischemic injuries ${ }^{9,10}$. We have also demonstrated that activation of beta II PKC ( $\beta$ IIPKC), but not other PKCs, contributes to heart failure pathophysiology in rodents ${ }^{11,12}$. Likewise, $\beta$ IIPKC activity is elevated in human failing hearts ${ }^{11}$. More recently, we provided evidence that a rationally designed peptide against $\beta \mathrm{IIPKC}, \beta \mathrm{II}_{\mathrm{V} 5-3}$, improves cardiac function in rats with hypertension-induced or post-myocardial infarctioninduced heart failure ${ }^{12,13}$. This peptide inhibits all $\beta$ IIPKC activities (referred to as a global $\beta$ IIPKC inhibitor in this paper).

Here, we use the global $\beta$ IIPKC inhibitor to identify mitofusin 1 (Mfn1) as a downstream $\beta$ IIPKC substrate involved in heart failure progression. Mfn1 is a ubiquitous and well-conserved GTPase responsible for regulating mitochondrial dynamics and bioenergetics. We show that $\beta$ IIPKC associates with Mfn 1 . Using a rationally designed peptide that selectively antagonizes Mfn1$\beta$ IIPKC association (SAM $\beta A$ ), we determine the contribution of Mfn1- $\beta$ IIPKC interaction and the resulting phosphorylation of Mfn1 to mitochondrial morphology and bioenergetics and to the pathology associated with heart failure. Our study provides evidence that inhibition of excessive Mfn1- $\beta$ IIPKC interaction and the resulting mitochondrial fragmentation and dysfunction are critical to heart failure-associated pathophysiology.

\section{Results}

$\beta$ IIPKC activation mediates mitochondrial fragmentation in heart failure. $\beta$ IIPKC activation contributes to heart failure pathophysiology ${ }^{14}$. However, the molecular mechanisms involved in this process, including the identification of critical substrates that are phosphorylated by this pleiotropic enzyme are not known. As we demonstrated before ${ }^{11}$, blocking protein-protein interaction between $\beta$ IIPKC and its anchor protein (RACK1) with $\beta \mathrm{II}_{\mathrm{V} 5-3}$ (the global inhibitor of $\beta \mathrm{IIPKC}^{15}$ ) improved isolated cardiomyocyte and whole heart contractility properties as well as cardiac remodeling in rats with myocardial infarction-induced heart failure (Fig. 1a-d; Supplementary Table 1). In these studies, rats were treated for six weeks with $\beta \mathrm{II}_{\mathrm{V} 5-3}$ at $3 \mathrm{mg}$ per $\mathrm{Kg}$ per day (a dose that we previously showed to be optimal ${ }^{11}$ ) in a sustained fashion using an Alzet pump implanted under the skin on the back of the animal. Treatment commenced after heart failure was established, 4 weeks after left descending coronary artery ligation to induce myocardial infarction (Fig. 1a). Control rats (sham group) were submitted to the same surgical procedure, but without left descending coronary artery ligation.

Using transmission electron microscopy, we noticed that the inhibition of global $\beta$ IIPKC activity had a prominent impact on cardiac mitochondrial number and size in heart failure (Fig. 1e, f). Failing hearts accumulated smaller and spherical (fragmented) mitochondria compared to the sham group, resulting in increased mitochondrial number to size ratio (Fig. 1e, f). These changes correlated with increased outer mitochondrial membrane proteins, but not the electron transport chain subunits, in failing hearts (Supplementary Figure 1a-c). Sustained inhibition of global $\beta \mathrm{IIPKC}$ activity using the $\beta \mathrm{II}_{\mathrm{V} 5-3}$ peptide at $3 \mathrm{mg}$ per $\mathrm{Kg}$ per day re-established mitochondrial number to size ratio in rats with heart failure as compared to sham rats (Fig. 1e, f). These findings demonstrate that $\beta$ IIPKC activation causes mitochondrial fragmentation in heart failure. Sustained global inhibition of $\beta I I P K C$ activity had no impact on mitochondrial DNA levels and markers of mitophagy in heart failure (Supplementary Figure 1a, d, e). Neither cardiac function nor mitochondrial number to size ratio were affected by chronic treatment with peptide $\beta \mathrm{II}_{\mathrm{V} 5-3}$ in Sham animals (Supplementary Figure 2).

Next, we demonstrated that only $\beta$ IIPKC among different PKC isozymes accumulated in the mitochondrial fraction in failing rat hearts (Fig. 1g, h). RACK1, the $\beta$ IIPKC anchoring protein ${ }^{16}$ also accumulated in the mitochondrial fraction of failing hearts (Fig. 1g, h). Moreover, blocking ßIIPKC-RACK1 interaction using $\beta \mathrm{II}_{\mathrm{V5-3}}$ peptide was sufficient to reduce $\beta \mathrm{IIPKC}$ levels in the mitochondrial fraction in heart failure (Fig. 1g, h). To determine whether $\beta$ IIPKC is located at the outer mitochondrial membrane or inside mitochondria, we incubated isolated cardiac mitochondria with proteinase $\mathrm{K}\left(5 \mu \mathrm{g} \mathrm{mL}^{-1}\right)$ for $15 \mathrm{~min}$ in isotonic mitochondrial buffer. Proteinase K completely degraded $\beta$ IIPKC and Tom20 (a protein located in the outer mitochondrial membrane), but not ALDH2 (an enzyme located in the matrix) (Fig. 1i). These findings demonstrated that, in failing rat hearts, $\beta I I P K C$ accumulates and binds to RACK1, as a result of $\beta$ IIPKC activation ${ }^{17}$, in the outer mitochondrial membrane.

Next, we validated our in vivo findings using neonatal cardiac myocyte cultures. Treatment of neonatal cardiac myocytes with angiotensin II for $4 \mathrm{~h}$ caused mitochondrial fragmentation, which was prevented by a concomitant treatment with the global $\beta \mathrm{IIPKC}$ inhibitor, $\beta \mathrm{II}_{\mathrm{V} 5-3}$ (Fig. $2 \mathrm{a}-\mathrm{c}$ ). The treatment also prevented mitochondrial accumulation of $\beta$ IIPKC that was induced by either angiotensin II or ceramide (Fig. 2d); these stressors are hallmarks of heart failure in humans ${ }^{18,19}$ that cause mitochondrial fragmentation in cardiomyocytes ${ }^{20,21}$.

To examine the functional importance of $\beta$ IIPKC on mitochondrial biology and cell survival, $\beta$ IIPKC was silenced using selective small interfering RNA (siRNA, Fig. 2e). Silencing $\beta$ IIPKC protected neonatal cardiac myocytes from $\mathrm{H}_{2} \mathrm{O}_{2}-$ mediated oxidative stress-induced metabolic dysfunction and cytotoxicity (Fig. 2f). $\beta \mathrm{II}_{\mathrm{V} 5-3}$ treatment and $\beta \mathrm{IIPKC}$ knockdown had a similar protective effect on neonatal cardiac myocytes, and the combination of genetic and pharmacological $\beta$ IIPKC suppression had no additional protection on neonatal cardiac myocyte metabolism and toxicity as compared with either single intervention (Fig. 2e, f). BIIPKC knockdown (with siRNA) or 

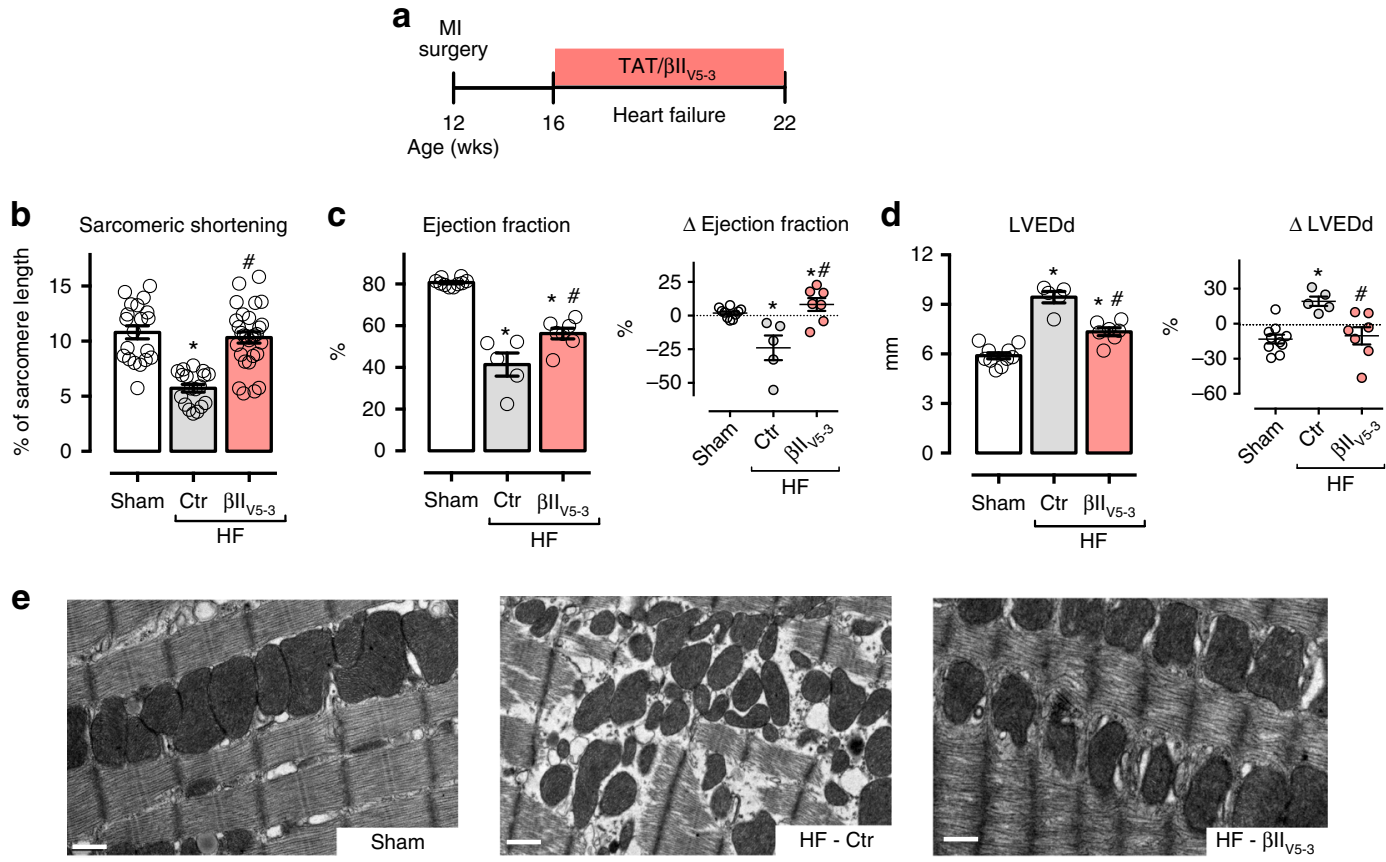

f
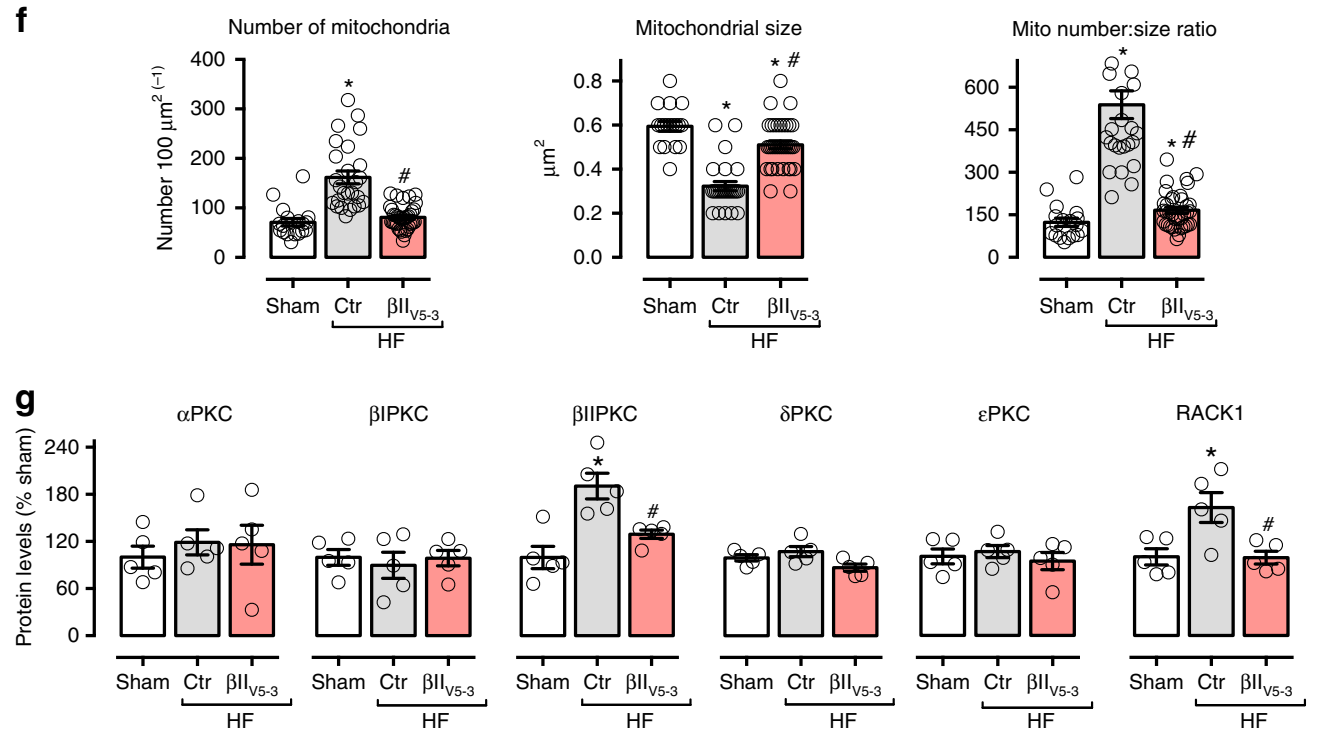

h

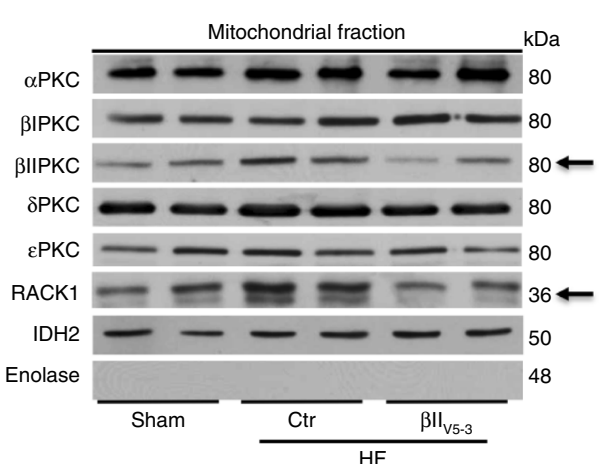

treatment with $\beta \mathrm{II}_{\mathrm{V} 5-3}$ also protected adult cardiomyocytes from oxidative stress (a known trigger of mitochondrial fragmentation $^{22}$ ) (Fig. 2g, h), similar to what we found in neonatal cardiomyocytes. These findings demonstrate that suppressing BIIPKC is sufficient to protect both neonatal and adult cardiac

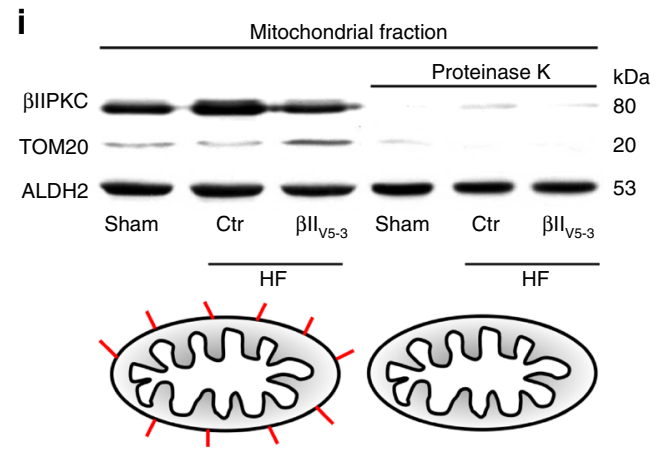

myocytes against oxidative stress-induced mitochondrial fragmentation and dysfunction.

We next determined whether $\beta$ IIPKC activation affects mitochondrial oxidative capacity in heart failure. To assess mitochondrial function, we measured oxygen consumption, 
Fig. 1 IIIPKC inhibition reduces mitochondrial fragmentation in heart failure. a Schematic panel of heart failure induction by myocardial infarction (MI) and the treatment protocol. Twelve-week-old male rats were subjected to Ml-induced heart failure by left anterior descending coronary artery permanent ligation. Four weeks after $\mathrm{MI}$ induction, rats were treated with either the global $\beta$ IIPKC-specific inhibitor $\left(\beta \|_{V 5-3}\right)$ or with a control peptide (TAT, used to deliver $\beta \|_{V 5-3}$ into the heart). Peptide treatment was continuous (for 6 weeks) using an Alzet pump, delivering at a rate of $3 \mathrm{mg}$ per $\mathrm{Kg}$ per day. b Sarcomeric shortening in isolated ventricular cardiomyocytes, c left ventricular ejection fraction and d LVEDd [left ventricular end-diastolic dimension] measured by echocardiography at the end of the experimental protocol, input: delta of measurements performed before and after treatment;

e representative cardiac transmission electron micrographs (scale bar: $0.5 \mu \mathrm{m}$ ); $\mathbf{f}$ quantification of intermyofibrillar mitochondrial number and area in the transmission electron micrographs; $\mathbf{g}$ PKCs and RACK1 protein levels in cardiac mitochondrial fraction and $\mathbf{h}$ representative western blots; and $\mathbf{i}$ representative western blots of mitochondrial $\beta \| \mathrm{IPKC}$ location in sham (white bars, $n=8)$, TAT-treated heart failure $(\mathrm{HF}-\mathrm{Ctr}$, gray bars, $n=5)$ and $\beta \|_{\mathrm{V} 5-3^{-}}$ treated heart failure $\left(\mathrm{HF}-\beta \|_{V 5-3}\right.$, red bars, $\left.n=7\right)$. Biochemical measurements were performed in the cardiac remote (viable) zone. Data are means \pm SEM ${ }^{\star} P<0.05$ vs. Sham rats. $\# P<0.05$ vs. HF-Ctr rats. One-way analysis of variance (ANOVA) with post-hoc testing by Duncan. For all the cardiac function studies, the observer was blinded to the experimental groups

absolute $\left(\mathrm{H}_{2} \mathrm{O}_{2}\right)$ and relative $\left(\mathrm{H}_{2} \mathrm{O}_{2}: \mathrm{O}_{2}\right)$ hydrogen peroxide release in isolated mitochondria from heart failure rats. Failing hearts displayed a significant reduction in the efficiency of mitochondrial oxidative phosphorylation compared to hearts from sham operated rats, as measured by respiratory control ratio (RCR, state 3: state 4; Fig. 3a). This response was mainly due to a reduction of State 3 respiratory rate (Fig. $3 \mathrm{~b}$ ) and basal respiration (State 2, Supplementary Figure 3a), while respiration in the absence of oxidative phosphorylation (State 4) was unaffected in these failing rat hearts (Supplementary Figure 3a). Moreover, these failing hearts had elevated mitochondrial State 2 (basal, Supplementary Figure 3a) and State 3 (oxygen consumption rate maximized by the addition of ADP, Fig. 3c) $\mathrm{H}_{2} \mathrm{O}_{2}$ release compared to hearts from the sham group. These changes were maximized when $\mathrm{H}_{2} \mathrm{O}_{2}$ was normalized by oxygen consumption rates (Fig. 3d, Supplementary Figure 3a), providing evidence of a tight connection between mitochondrial oxygen consumption and reactive oxygen species release in heart failure.

We next determined the effect of the global $\beta I I P K C$ inhibition on mitochondrial bioenergetics in heart failure. Sustained inhibition of $\beta \mathrm{IIPKC}$ activity with $\beta \mathrm{II}_{\mathrm{V} 5-3}$ improved the efficiency of mitochondrial oxidative phosphorylation (Fig. 3a), mainly by preserving State 3 respiratory rate in heart failure (Fig. 3b). Moreover, mitochondria isolated from heart failure animals treated with the global $\beta$ IIPKC inhibitor displayed reduced $\mathrm{H}_{2} \mathrm{O}_{2}$ and $\mathrm{H}_{2} \mathrm{O}_{2}: \mathrm{O}_{2}$ release when compared to TAT-treated (control) heart failure animals (Fig. 3c, d). These findings suggest that inhibiting BIIPKC accumulation in the outer mitochondrial membrane is associated with the increase of mitochondrial oxygen consumption and reduced excessive mitochondrial $\mathrm{H}_{2} \mathrm{O}_{2}$ release in rats with heart failure. Next, we determined how $\beta I I P K C$ affects mitochondrial biology in heart failure.

$\beta$ IIPKC interacts with and inhibits Mfn1 activity. Mitochondrial number and size are tightly regulated by fusion and fission, in a process termed mitochondrial dynamics. Because heart failure is associated with excessive mitochondrial fragmentation, we hypothesized that elevated $\beta$ IIPKC levels on the outer mitochondrial membrane resulted in phosphorylation of proteins that regulate fusion or fission. We first determined the levels and activity of the main GTPases involved in mitochondrial dynamics in the heart. Only the levels of Mfn1 (a mitochondrial fusionrelated enzyme) were elevated in cardiac mitochondria during heart failure (Fig. 3e), yet its GTPase activity was significantly reduced as compared to the sham group (Fig. 3f), suggesting accumulation of dysfunctional $\mathrm{Mfn} 1$ in heart failure. $\beta$ IIPKC inhibition by $\beta \mathrm{II}_{\mathrm{V} 5-3}$ selectively re-established cardiac Mfn1 levels and activity in heart failure, without affecting the level or activity of mitofusin 2 (Mfn2, Fig. 3e, f).

Given that $\beta$ IIPKC and Mfn1 accumulated on the outer mitochondria membrane during heart failure, and that blocking global $\beta$ IIPKC activity reduced mitochondrial Mfn1 levels and reestablished the mitochondrial number to size ratio, we next determined whether $\beta$ IIPKC and Mfn1 form a complex. Analysis of anti-Mfn1 immunoprecipitates by immunoblotting with antiBIIPKC antibody demonstrated an increased association of BIIPKC with Mfn1 in cardiac mitochondria isolated from rats with heart failure (Fig. 3g, top panel). In reciprocal experiments, immunoblot analysis of anti-ßIIPKC immunoprecipitates with anti-Mfn1 antibody confirmed the interaction (Fig. 3g, middle panel). No change in cardiac $\beta$ IIPKC-Mfn2 interaction was seen in heart failure (Supplementary Figure 1f). Confirming a role for Mfn1 in BIIPKC association with the mitochondria, in Mfn1-null MEFs (mouse embryonic fibroblasts) mitochondrial $\beta I I P K C$ levels were lower as compared to WT MEFs (Fig. 3h). However, Mfn1 is not essential for mitochondrial accumulation of $\beta$ IIPKC upon stimulation with $10 \mathrm{nM}$ phorbol ester 12-myristate 13acetate (PMA, an activator of most PKC isozymes ${ }^{11}$ ).

Next, we determined the functional impact of $\beta$ IIPKC on Mfn1. Immunoblot analysis of anti-Mfn1 immunoprecipitates with anti-serine/threonine antibody revealed an increased phosphorylation at the molecular size of Mfn1 in heart failure samples (Fig. $3 \mathrm{~g}$, middle panel) that was reduced by inhibiting $\beta \mathrm{IIPKC}$ activity with $\beta \mathrm{II}_{\mathrm{V} 5-3}$. Co-immunoprecipitation experiments demonstrated that recombinant $\beta$ IIPKC interacts with recombinant Mfn1 (Fig. 4a) and active $\beta$ IIPKC (with PS/DG/ $\mathrm{Ca}^{+2}$ ), but not inactive $\beta$ IIPKC or its alternative splicing $\beta I P K C$, phosphorylated Mfn1 [measured by incorporation of radiolabeled $\mathrm{P}^{32}$-ATP] (Fig. 4b). Finally, as expected from our findings in whole hearts, active recombinant $\beta$ IIPKC (and not active $\beta$ IPKC) significantly reduced the GTPase activity of the recombinant Mfn1 in this in vitro assay (Fig. 4c).

Mass spectrometry analysis of the in vitro phosphorylation products identified S86, S284, and S290 in the Mfn1 GTPase domain to be the $\beta$ IIPKC phosphorylated sites (Fig. 4d). A serineto-alanine substitution demonstrated that S86A, but not S284A or S290A, was sufficient to protect MEFs against $\beta$ IIPKC activationinduced cytotoxicity (Fig. 4e), suggesting S86 as a critical site to induce $\beta$ IIPKC-mediated Mfn1 inactivation. Mfn1 $\mathrm{S} 86$ is conserved in many species and it is not present in Mfn2 (another critical mitofusin involved in mitochondrial dynamics; Fig. 4d). Collectively, these findings suggest that phosphorylation of Mfn1 by $\beta$ IIPKC at S86 inhibits its GTPase activity and increases cytotoxicity.

Design of selective inhibitor of Mfn1- $\beta$ IIPKC association. Our in vivo and in vitro data provide evidence that Mfn1 phosphorylation and consequent inhibition of its GTPase activity by $\beta I I P K C$ is associated with impaired mitochondrial morphology and oxidative capacity. However, in addition to Mfn1, $\beta$ IIPKC phosphorylates many other substrates. To determine whether inhibition of Mfn1- $\beta$ IIPKC interaction alone is sufficient to 
Neonatal cardiomyocytes

a
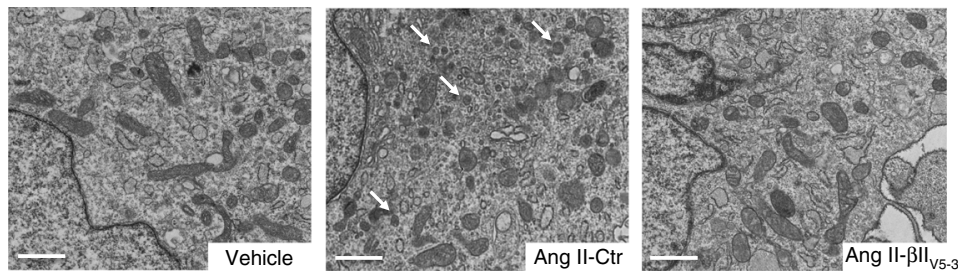

b
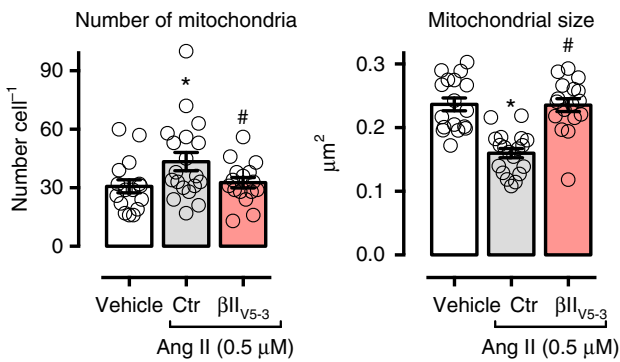

C
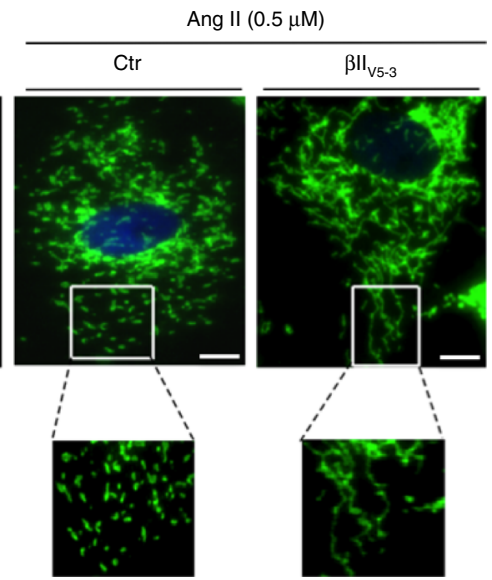
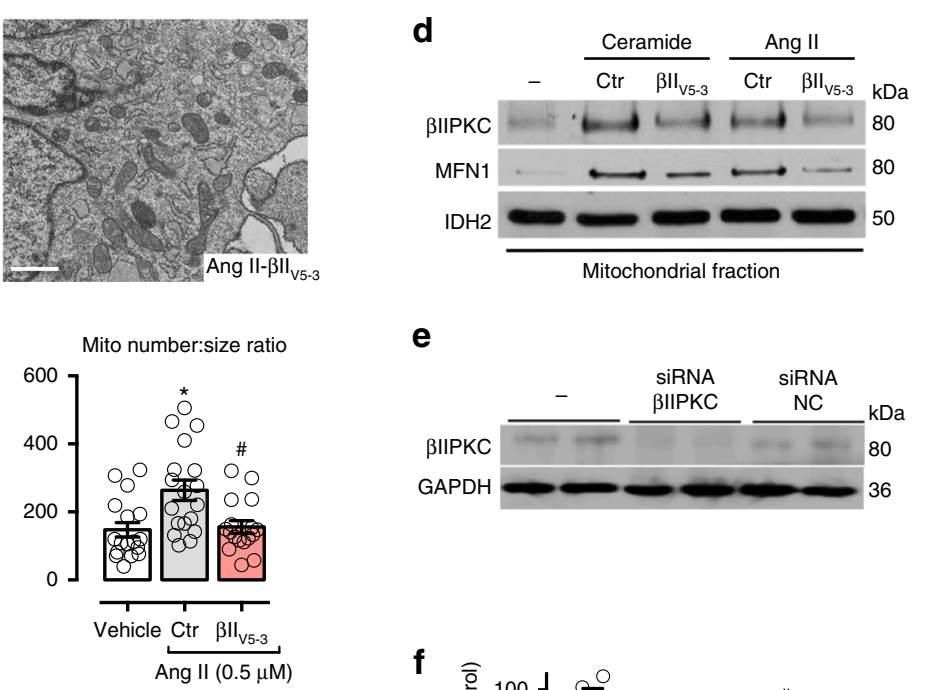

e
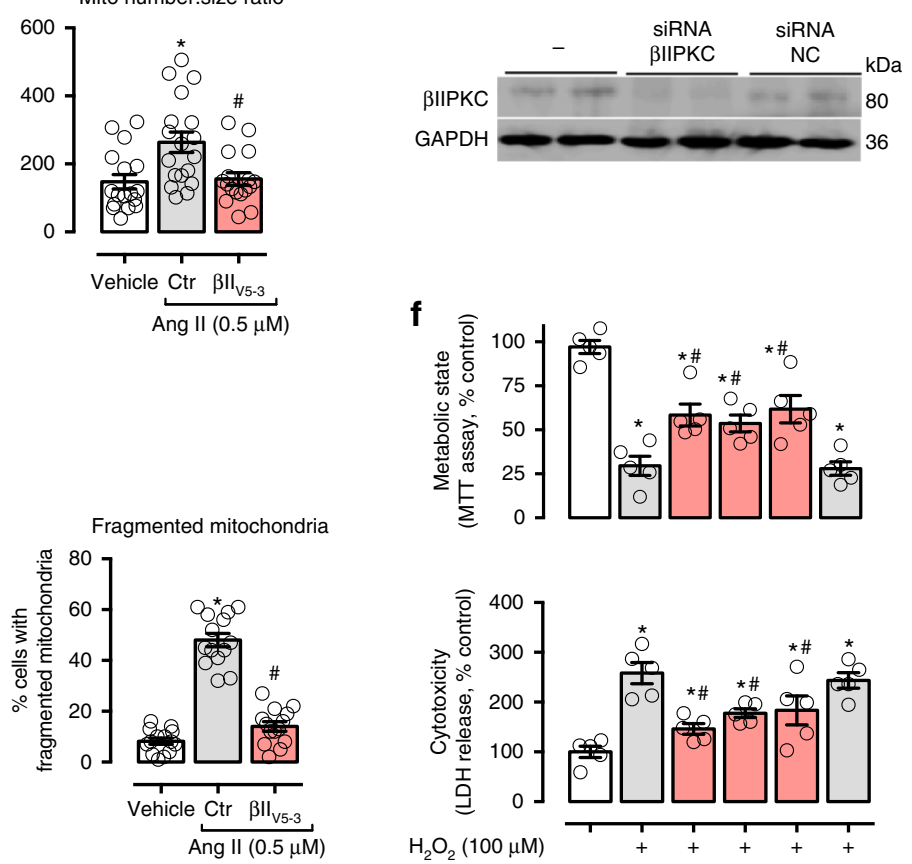
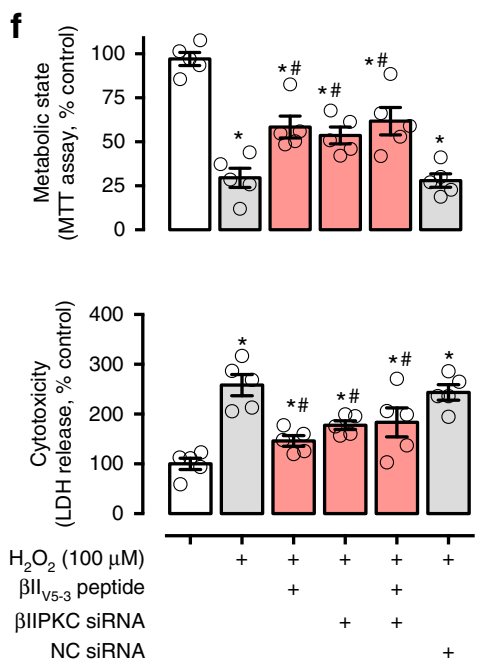

Adult cardiomyocytes
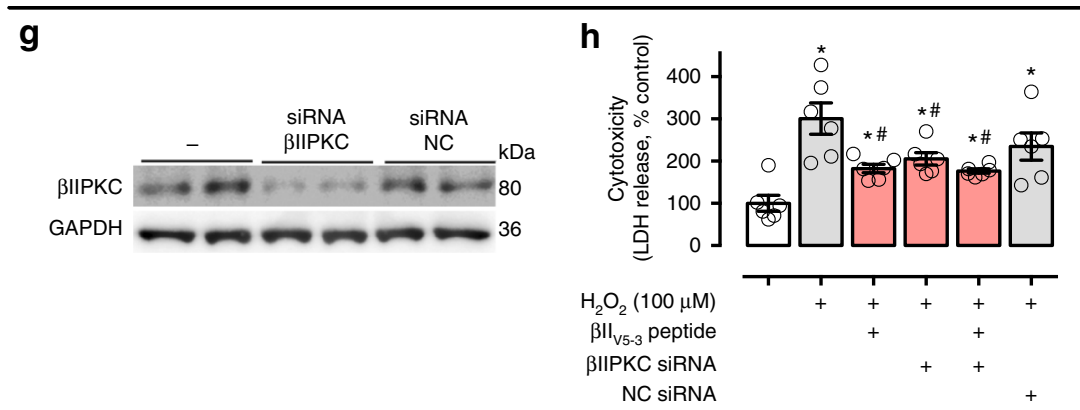

Fig. 2 BIIPKC inhibition reduces mitochondrial fragmentation in cultured cardiomyocytes. a Representative transmission electron micrographs (white arrows indicate small mitochondria, scale bar: $1 \mu \mathrm{m}$ ) and $\mathbf{b}$ quantification of mitochondrial number and size in the transmission electron micrographs of neonatal rat cardiomyocytes in culture treated with TAT (carrier) or $\beta I_{V 5-3}$ peptides for 30 min followed by incubation with angiotensin II ( $0.5 \mu \mathrm{M}$ for $4 \mathrm{~h}$, $n=5$ per group). c Cells were then stained with anti-Tom20 antibody (green) and Hoechst stain and counted. Mitochondrial morphology was analyzed using a $63 x$ oil immersion lens (scale bar: $5 \mu \mathrm{m}$ ). The analysis was done in a blinded fashion. The boxed area in each upper panel is enlarged under each micrograph. d Neonatal rat cardiomyocytes in culture were treated with TAT or $\beta \|_{V 5-3}$ peptides for 30 min followed by incubation with either $C 2$-ceramide $(40 \mu \mathrm{M})$ or angiotensin II $(0.5 \mu \mathrm{M})$ for $4 \mathrm{~h}$. After incubation, the mitochondrial levels of $\beta$ IIPKC and Mfn1 were detected using specific antibodies (representative blot of three independent experiments). e Neonatal rat cardiomyocytes in culture were transfected with $\beta I I P K C$ silence RNA ( $\beta I I P K C$ siRNA) or control (NC-siRNA). f $48 \mathrm{~h}$ later cells were treated with TAT or $\beta \mathrm{Il}_{\mathrm{V} 5-3}$ peptides for $30 \mathrm{~min}$ followed by incubation with $\mathrm{H}_{2} \mathrm{O}_{2}(100 \mu \mathrm{M}, 24 \mathrm{~h}$ ). After incubation, cell viability and toxicity were measured by MTT assay and LDH release, respectively. $\mathbf{g}$ Adult rat cardiomyocytes in culture were transfected with $\beta$ IIPKC-siRNA or NC-siRNA ( $n=6$ per group). $\mathbf{h} 24 \mathrm{~h}$ later cells were treated with TAT or $\beta \|_{V 5-3}$ peptides for 30 min followed by incubation with $\mathrm{H}_{2} \mathrm{O}_{2}(100 \mu \mathrm{M}, 1 \mathrm{~h})$. Cell toxicity was measured by $L D H$ release. Data are means $\pm \mathrm{SEM}$. ${ }^{\star} P<0.05$ vs. control cells. \#P<0.05 vs. Ang II- or $\mathrm{H}_{2} \mathrm{O}_{2}$-treated cells. One-way analysis of variance (ANOVA) with post-hoc testing by Duncan 
a

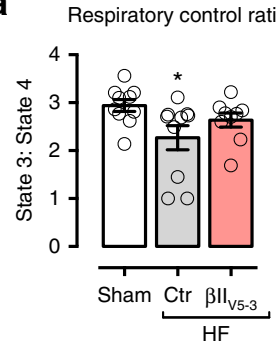

b

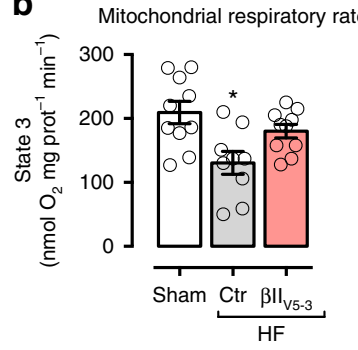

C

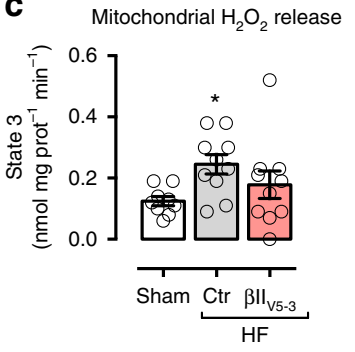

d Mitochondrial $\mathrm{H}_{2} \mathrm{O}_{2}: \mathrm{O}_{2}$

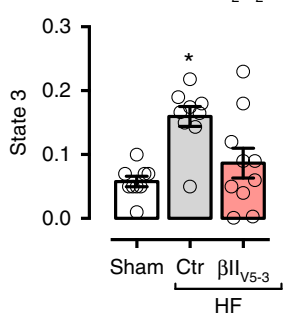

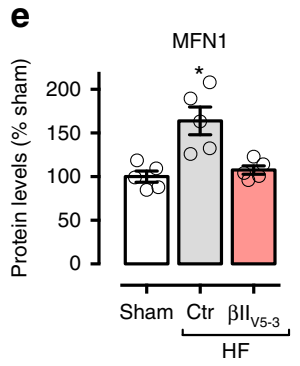

f

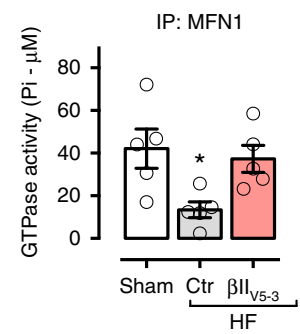

MFN2
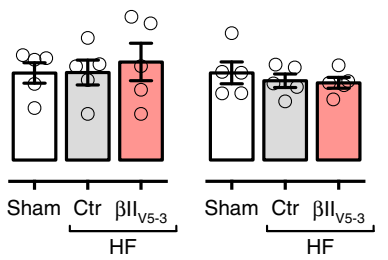

FIS1

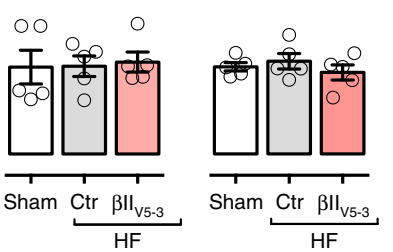

g

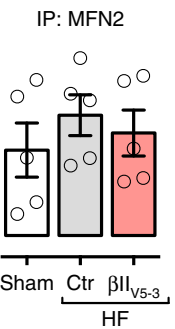

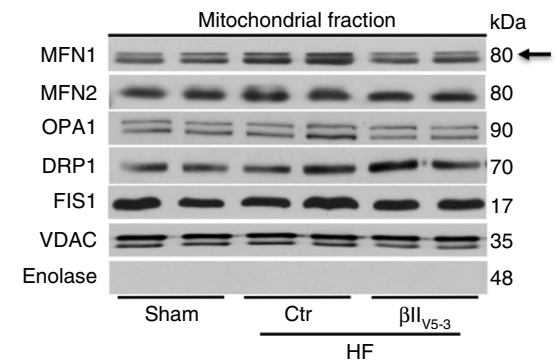

h

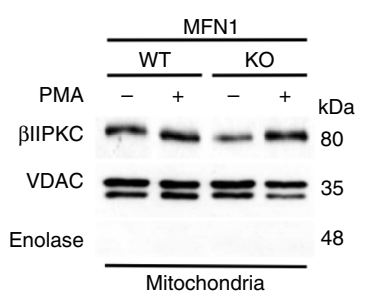

Fig. 3 BIIPKC inhibition improves bioenergetics in failing hearts. a Mitochondrial respiratory control ratio, b State 3-dependent oxygen control rate, c absolute $\mathrm{H}_{2} \mathrm{O}_{2}$ release and $\mathbf{d} \mathrm{H}_{2} \mathrm{O}_{2} \mathrm{O}_{2}$ in heart samples from sham (white bars, $n=10$ ), TAT-treated heart failure $(\mathrm{HF}-\mathrm{Ctr}, \mathrm{gray}$ bars, $n=10)$ and $\beta \mathrm{II}_{V 5-3^{-}}$ treated heart failure (HF- $\beta \|_{V 5-3}$, red bars, $\left.n=10\right)$. e Mfn1, Mfn2, Opa1, Drp1, and Fis1 protein levels and representative western blots; and $\mathbf{f}$ Mfn1 and Mfn2 GTPase activity in cardiac mitochondrial fraction ( $n=5$ per group) from sham, HF-Ctr and HF- $\beta \|_{V 5-3}$. $\mathbf{g}$ Cardiac mitochondrial Mfn1 and $\beta \| \mathrm{PKC}$ immunoprecipitate probed against anti-ßIIPKC, Mfn1 and phosphorylated serine/threonine antibodies (representative blot of three independent experiments) from sham, HF-Ctr and HF- $\beta I_{V 5-3}$ groups. Biochemical measurements were performed in the cardiac remote (viable) zone. These measurements were performed at the end of the experimental protocol. $\mathbf{h}$ Mfn1 wild type (WT, $n=5)$ and knockout $(K O, n=5)$ MEFs were treated with PMA [10 nM phorbol ester 12-myristate 13-acetate, for $30 \mathrm{~min}$, an activator of most PKC isozymes ${ }^{11}$ ] and accumulation of mitochondrial $\beta$ IIPKC was analyzed by western blot (representative blots of three independent experiments). Data are means \pm SEM. ${ }^{\star} P<0.05$ vs. Sham rats. One-way analysis of variance (ANOVA) with post-hoc testing by Duncan

prevent accumulation of fragmented and dysfunctional mitochondria upon stress, we developed a peptide that selectively antagonizes Mfn1- $\beta$ IIPKC association, termed SAM $\beta$ A.

We have previously shown that two proteins that interact usually share short sequences of homology that represent sites of both inter- and intra-molecular interactions ${ }^{17,23,24}$. More recently, we described a similar rational approach to develop PKC inhibitors that selectively block interaction and phosphorylation of only one protein substrate of these multi-substrate kinases $6,7,25$. Using the same approach, we rationally designed $S A M \beta A$. First, we used sequence alignment software ${ }^{26}$ to identify Mfn1-like sequence in $\beta$ IIPKC (Fig. 5a).

The $\beta$ IIPKC/Mfn1 homologous sequences, highlighted in color in Fig. $5 \mathrm{a}$ in the crystal structure of Mfn1 (5GOM) and $\beta$ PKC (3PFQ), appear to be exposed $\alpha$-helixes in the proteins and thus, likely accessible for protein-protein interaction (Fig. 5b). To help stabilize the $\alpha$-helix structure, we added two amino acids at the Cterminus of each peptide. We reasoned that if these sequences are important for the protein-protein interaction between $\beta I I P K C$ and Mfn1, they should be conserved across species. Indeed, RNAENFDRF is conserved in $\beta$ IIPKC, but not in its alternative splicing $\beta I P K C$ (Fig. 5c, d). Conversely, NELENFTKQ is conserved in Mfn1, but not in the homologous Mfn2 protein (Fig. 5e, f). A BLAST search of the human genome identified four other proteins with homologous sequences. However, these sequences were not evolutionarily conserved (Fig. 5g; Supplementary Table 2), except in aPKC (Fig. 5g). However, mitochondrial aPKC levels were not changed in failing hearts compared to sham animals (Fig. 1g). Together these data suggest a specific role for RNAENFDRF for BIIPKC and Mfn1 protein-protein interaction.

Next, we synthesized the peptide RNAENFDRF (SAM $\beta A$ ) and conjugated it to the cell penetrating TAT protein-derived peptide, $\mathrm{TAT}_{47-57}{ }^{27}$, and determined its effect in protecting MEFs against oxidative stress-induced cytotoxicity. Four other peptides related to protein-protein interaction derived from either $\beta$ IIPKC or Mfn1 were used as controls (P251-254, Supplementary Figure 4). Oxidative stress was induced by treating cells with $\mathrm{H}_{2} \mathrm{O}_{2}$. Only SAM $\beta A$ (derived from $\beta$ IIPKC), but not P255, derived from Mfn1, or P251-254 control peptides, was effective in mitigating oxidative stress-induced cytotoxicity (Fig. 5h, i upper panel). SAM $\beta$ A also protected WT MEFs, but not Mfn1 knockout MEFs, 
a
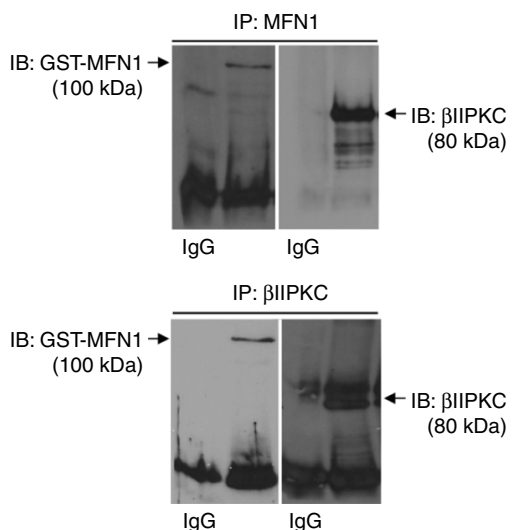

d
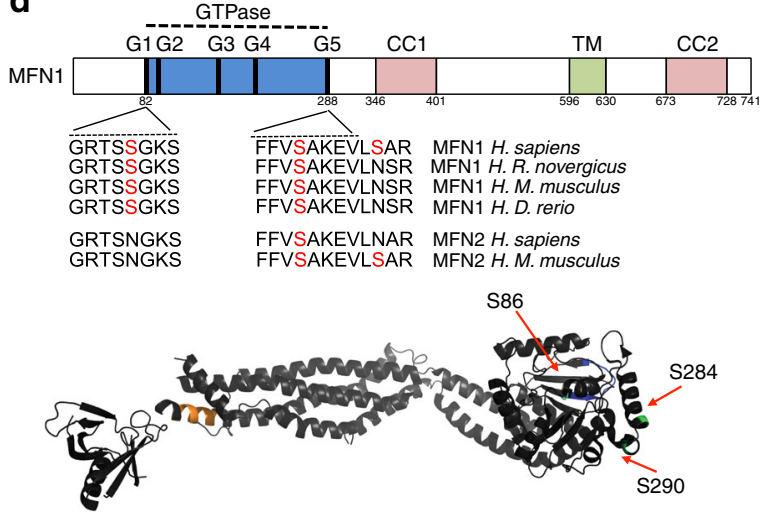

b

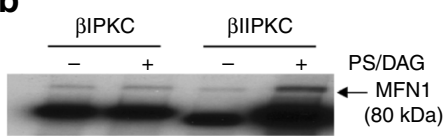

C

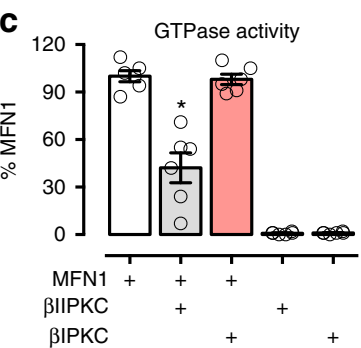

e
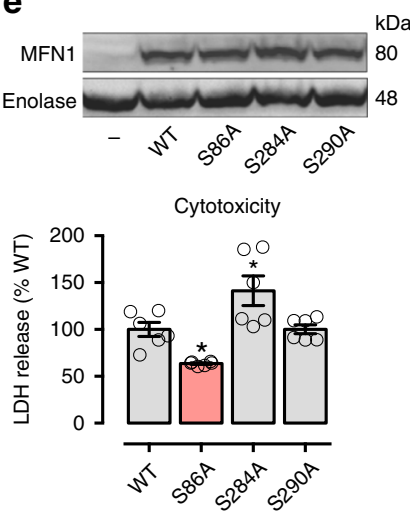

Fig. 4 Characterization of Mfn1- $\beta$ IIPKC protein-protein interaction. Recombinant Mfn1 (GST-Mfn1, 25 ng) was incubated with recombinant $\beta$ IIPKC (GST$\beta$ IIPKC, $25 \mathrm{ng}$ ) in the presence and absence of phosphatidylserine and diacylglycerol (PS/DAG/Ca ${ }^{2+}, \mathrm{PKC}$ activators) for $30 \mathrm{~min}$ at $37^{\circ} \mathrm{C}$. a Coimmunoprecipitates with anti-Mfn1 and $\beta$ IIPKC were analyzed by western blot. b Mfn1 phosphorylation was evaluated using radioactive [32P] ${ }^{32 P-A T P}$ incorporation after incubation with either $\beta$ IIPKC or its alternative splicing $\beta I P K C$ in the presence and absence of classic PKC activators. Representative blots of three independent experiments. c GTPase activity of Mfn1 determined in the presence of active $\beta$ IIPKC or its alternative splicing $\beta$ IPKC ( $n=6$ per group). $\mathbf{d}$ Conservation of the $\beta$ IIPKC phosphorylation in human orthologs of Mfn1 in rat, mouse, and zebrafish and Mfn2 in mouse. Ribbon presentation of the 3D structure of Mfn1 phosphorylation at the $\beta$ IIPKC site (PDB: 5GOM). e Individual replacement of serine by alanine at residues 86,284 , and 290 in Mfn1 knockout MEFs. Cells were transfected with the following constructs: WT, S86A, S284A and S290A. $48 \mathrm{~h}$ after transfection cells were incubated with $\mathrm{H}_{2} \mathrm{O}_{2}(100 \mu \mathrm{M}, 24 \mathrm{~h})$ and measured cytotoxicity (LDH release, $n=6$ per group). Data are means $\pm \mathrm{SEM}$. ${ }^{\star} P<0.05$ vs. control. One-way analysis of variance (ANOVA) with post-hoc testing by Duncan

from antimycin $\mathrm{A}$, an inhibitor of the mitochondrial electron transport chain (Fig. 5h, i lower panel).

Given that mitochondrial Mfn1 phosphorylation and inhibition by $\beta$ IIPKC is associated with accumulation of fragmented/ dysfunctional mitochondria and oxidative stress in failing hearts, we next determined the effect of the SAM $\beta A$ peptide on mitochondrial morphology in cardiac myocytes. Treatment of cultured neonatal cardiac myocytes with SAM $\beta A$ prevented mitochondrial fragmentation triggered by angiotensin II (Fig. 5j). SAM $\beta$ A reduced the excessive association of $\beta$ IIPKC with Mfn1 induced by angiotensin II in cardiac myocytes, as measured by co-immunoprecipitation (Fig. 5k). Finally, the treatment of cardiac neonatal myocytes with SAM $\beta A$ had a better effect in protecting against $\mathrm{H}_{2} \mathrm{O}_{2}$-induced metabolic dysfunction when compared to the global $\beta \mathrm{IIPKC}$ inhibitor, $\beta \mathrm{II}_{\mathrm{V} 5-3}$ (Fig. 5l).

SAM $\beta A$ treatment improves heart failure outcome in rats. To investigate the contribution of Mfn1-BIIPKC interaction to the pathophysiology of heart failure, we delivered the SAM $\beta A$ peptide (or its control peptide, TAT) to rats with myocardial infarctioninduced heart failure; the peptides were delivered in a sustained fashion at $3 \mathrm{mg}$ per $\mathrm{kg}$ per day, using subcutaneously implanted
Alzet pumps. Treatments were commenced 4 weeks after myocardial infarction and lasted 6 weeks (from 4 to 10 after inducing myocardial infarction; Fig. 6a). This 6-week treatment with SAM $\beta A$ resulted in a significant increase of cardiac function (measured by ventricular ejection fraction) compared to TATtreated (control) heart failure animals (Fig. 6b). SAM $\beta A$ treatment also decreased left ventricular end-diastolic diameter (LVEDd) as compared to control heart failure group (Fig. 6c; Supplementary Table 3). The better cardiac function following SAM $\beta A$ treatment was likely due to improved integrity of cardiac myofibril and mitochondria structures, as evidenced by transmission electron microscopy (Fig. 6d); sustained SAM $\beta A$ treatment prevented the accumulation of smaller and spherical (fragmented) mitochondria seen in the control rats with heart failure. Sustained SAM $\beta A$ delivery had no impact on blood pressure in rats with heart failure (Supplementary Table 3).

Sustained inhibition of Mfn1- $\beta$ IIPKC interaction using $S A M \beta A$ re-established mitochondrial number to size ratio in heart failure compared to sham group (Fig. 6d, e). Six weeks of treatment with $\mathrm{SAM} \beta \mathrm{A}$ also improved mitochondrial oxidative phosphorylation efficacy in heart failure (Fig. 6f), mainly by preserving State 2 (Supplementary Figure $3 \mathrm{~b}$ ) and State 3 respiratory rates (Fig. $6 \mathrm{~g}$ ). In addition, rats with established heart 
a Human $\beta$ IIPKC Human MFN1
N - A E N F 629 (SAMBA) NELEN F 729 (P255)

* $\quad * *$
C Human BIIPKC RíNAEN FID R F632 Rat $\quad$ IIIPKC RINAEN FID R F632 Mouse $\quad$ BIIPKC RINAEN F D R632 Chicken BIIPKC RiNAENFD R F631 $\star \star \star \star \star * \star * \star * \star$ e Human Rat Mouse Chicken MFN1 NELEN FT K Q 733 MFN1 RIELEN FS K 0733 MFN1 S:ELEN F S K Q 733 MFN1 NELDRFIT K H 733 $\star \star \wedge \wedge \star \wedge \star$

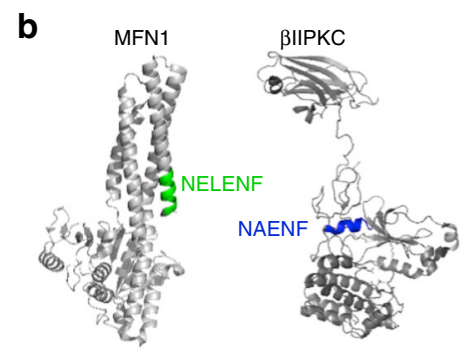

h
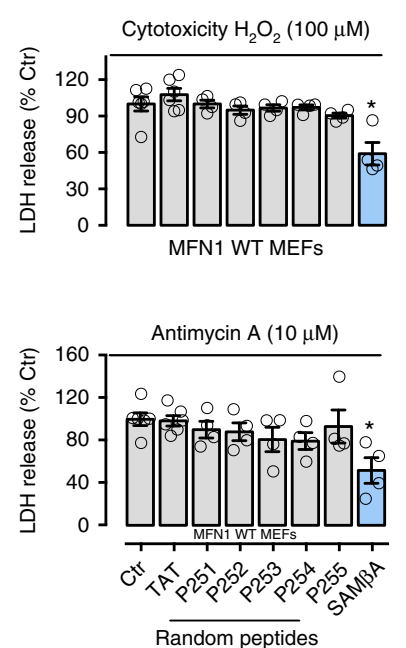

d

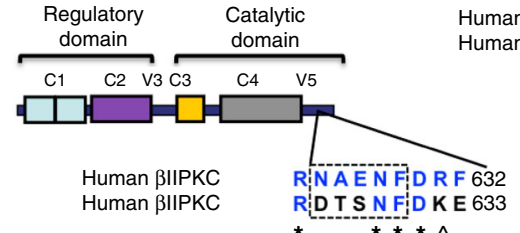

RTS TSERE 633 $* * * \wedge$ $f$

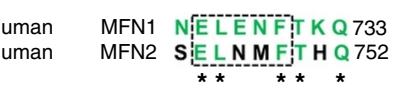

g

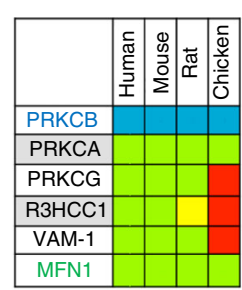

All amino acids identicalsimilar 6-8/9 amino acids identical/similar 1-5/9 amino acids identical/similar

No protein or homology at al i

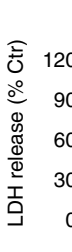

Cytotoxicity $\mathrm{H}_{2} \mathrm{O}_{2}(100 \mu \mathrm{M})$

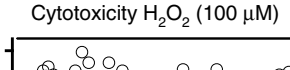

j

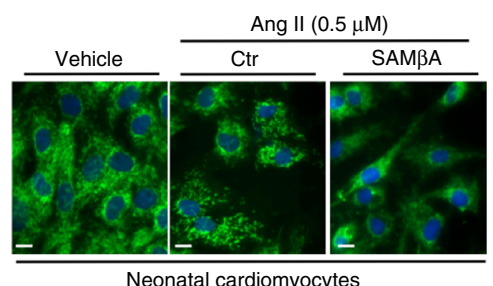
MFN1 KO MEFs

Neonatal cardiomyocytes

k

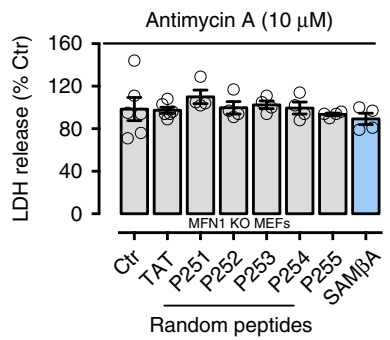

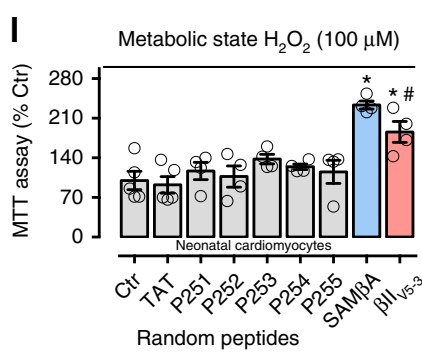

Fig. 5 Rational design of peptide that selectively inhibits Mfn1 and $\beta I I P K C$ interaction. a Sequence alignment of human $\beta I I P K C$ and Mfn1 identified a short sequence of homology, RNAENF/NELENF. b NELENF in Mfn1 (PDB: 5GOM) and RNAENF in the V5 domain of $\beta$ IIPKC (PDB: 3PFQ) are exposed and available for protein-protein interactions (see colored structures). Note that the alternatively spliced $\beta I P K C$ varies from $\beta I I P K C$ only in the $V 5$ domain ${ }^{15}$. c Conservation of RNAENFDRF sequence in $\beta I I P K C$ in a variety of species. d RNAENFDRF sequence is not present in the V 5 domain of $\beta I P K C$, a $\beta I I P K C$ alternative spliced form. e Conservation of NELENFTKQ in Mfn1 in a variety of species. $\mathbf{f}$ NELENFTKQ sequence is not present in Mfn2. $\mathbf{g}$ RNAENFDRF sequence is found in 6 human proteins. Heat-map of the RNAENFDRF conservation in orthologous of these proteins shows RNAENFDRF conservation only in $\beta$ IIPKC. (Further information about all the proteins is given in the Supplementary Table 2). *Denotes identity, and ^ denotes homology. $\mathbf{h}$ Mfn1 wild type (WT, $n=4-6$ ) and $\mathbf{i}$ Mfn1 knockout (KO, $n=4-6$ ) MEFs were treated with TAT, p251-255 or SAM $\beta$ A peptides for 30 min followed by incubation with either $\mathrm{H}_{2} \mathrm{O}_{2}(100 \mu \mathrm{M}, 24 \mathrm{~h})$ or Antimycin $\mathrm{A}(10 \mu \mathrm{M}, 24 \mathrm{~h})$. After incubation, cytotoxicity was measured by LDH release. $\mathbf{j}$ Neonatal rat cardiomyocytes in culture were treated with TAT or SAM $\beta A$ for $30 \mathrm{~min}$ followed by incubation with angiotensin II ( $0.5 \mu \mathrm{M}$ for $4 \mathrm{~h}, n=5$ per group). Cells were then stained with anti-Tom20 antibody (green) and Hoechst stain and counted. Mitochondrial morphology was analyzed using a $\times 63$ oil immersion lens (scale bar: 10 $\mu \mathrm{m})$. $\mathbf{k}$ Co-immunoprecipitates with anti- $\beta$ IIPKC were analyzed in the mitochondrial fraction by western blot (representative blots of three independent experiments). I Neonatal rat cardiomyocytes in culture were treated with TAT, P251-255, SAM $\beta A$ or $\beta I_{V 5-3}$ peptides for 30 min followed by incubation with $\mathrm{H}_{2} \mathrm{O}_{2}$ (100 $\mu \mathrm{M}$ for $24 \mathrm{~h}, n=4-5$ per group). After incubation, cell viability was measured by MTT assay. Data are means $\pm \mathrm{SEM}$. ${ }^{\star} P<0.05$ vs. Ctr. \#P $<0.05$ vs. SAM $\beta A$-treated cells. One-way analysis of variance (ANOVA) with post-hoc testing by Duncan

failure that were treated with the SAM $\beta A$ for 6 weeks presented reduced cardiac mitochondrial $\mathrm{H}_{2} \mathrm{O}_{2}$ and $\mathrm{H}_{2} \mathrm{O}_{2}: \mathrm{O}_{2}$ release when compared to control heart failure rats (Fig. $6 \mathrm{~h}$, i; Supplementary Figure $3 \mathrm{~b}$ ). Of interest, sustained SAM $\beta A$ treatment was sufficient to reduce apoptotic cell death in failing hearts as compared to that in hearts from vehicle-treated rats (Supplementary Figure 3c). Therefore, sustained blockage of Mfn1- $\beta$ IIPKC interaction was critical to protect against accumulation of fragmented/dysfunctional mitochondria with an impact on cell death in heart failure.

Next, we tested the effectiveness of SAM $\beta A$ on reducing Mfn1$\beta$ IIPKC interaction in failing hearts. Immunoblot analysis of antiBIIPKC immunoprecipitates with anti-Mfnl and anti-serine/ threonine antibodies demonstrated increased Mfn1- $\beta$ IIPKC association and phosphorylation at the molecular size of Mfn1 in heart failure samples that were reduced by SAM $\beta$ A (Fig. 6j, left panel).
The selectivity of SAM $\beta A$ as a Mfn1- $\beta$ IIPKC association and phosphorylation inhibitor was also tested by determining its impact on BIIPKC-troponin I association. Troponin I is a well-known $\beta$ IIPKC substrate in heart failure ${ }^{28}$. Immunoblot analysis of antiBIIPKC immunoprecipitates with anti-troponin I and antiphosphorylated troponin I antibodies demonstrated an elevated BIIPKC-troponin I interaction and phosphorylation at the molecular size of troponin I in heart failure samples that were not affected by SAM $\beta A$ (Fig. 6j, right panel). These findings provide evidence that SAM $\beta$ A selectively targets the elevated Mfn1- $\beta$ IIPKC interaction in heart failure and not another $\beta I I P K C$ substrate.

Finally, we compared the benefit of treatments with $\beta \mathrm{II}_{\mathrm{V} 5-3}$ (a global $\beta$ IIPKC inhibitor) relative to SAM $\beta A$ (a selective Mfn1$\beta$ IIPKC protein-protein interaction inhibitor) in improving ventricular function and remodeling in heart failure. Both 
a

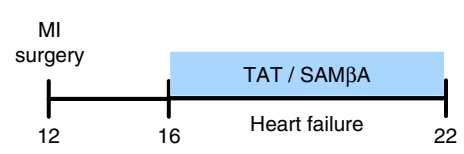

Age (wks)

b

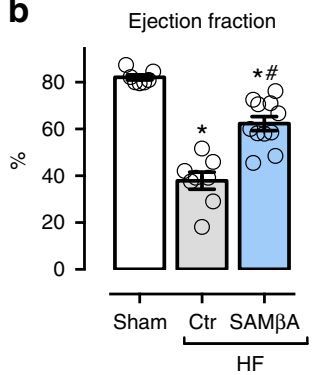

d
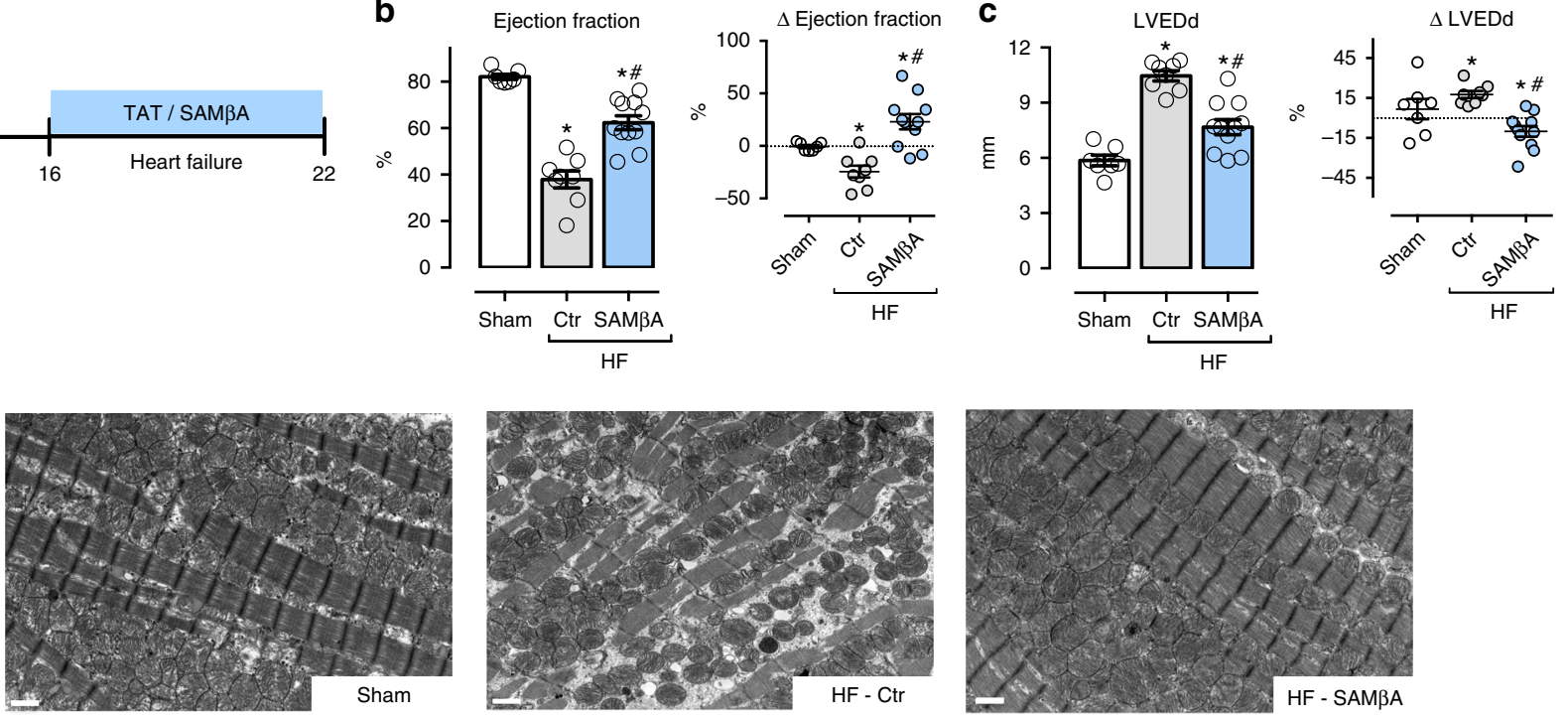

e
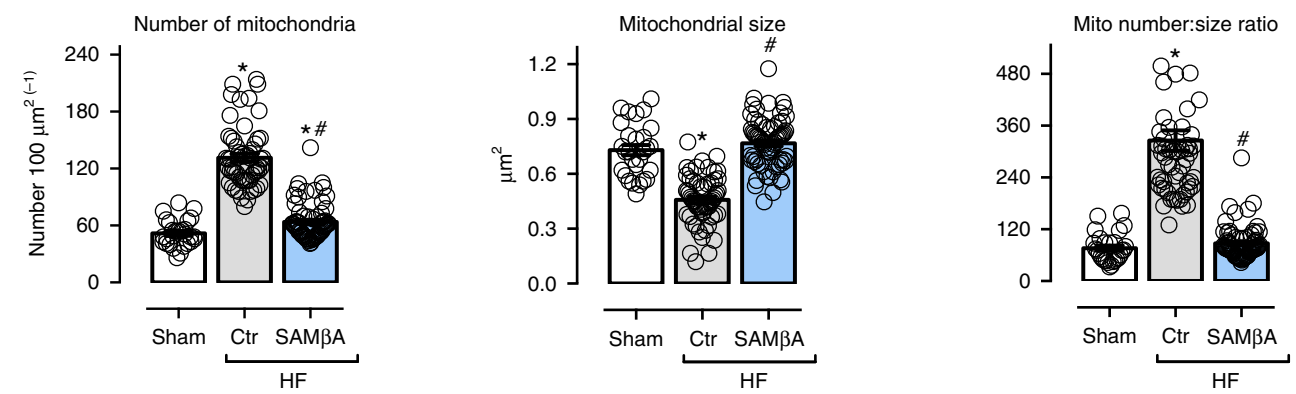

$\mathbf{f}$

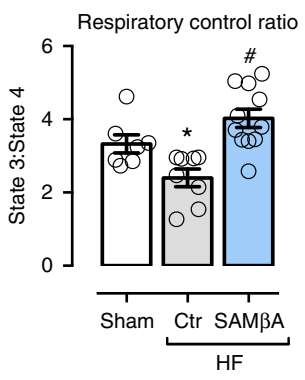

g Mitochondrial respiratory rate

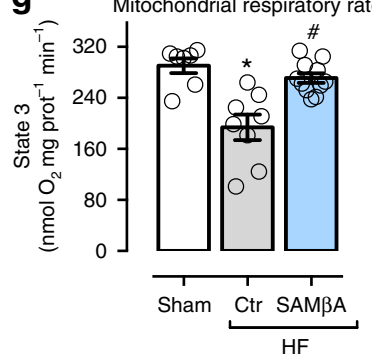

j

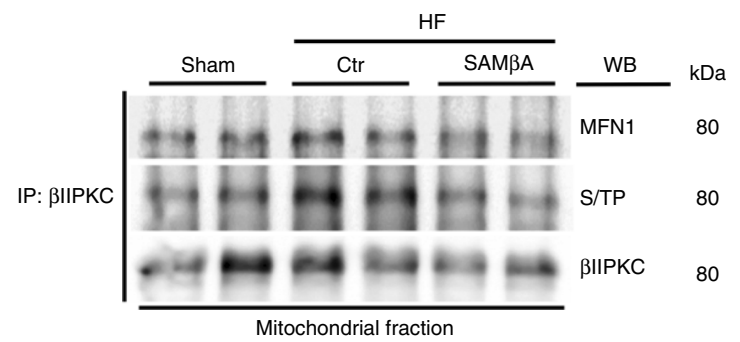

treatments prevented the progression of heart failure, measured by the drop in left ventricular ejection fraction and increase in cardiac dilation over time (before vs. after treatment, Fig. 7a, b). However, only the treatment with $S A M \beta A$ resulted in a significant improvement in left ventricular ejection fraction when compared to week 4 (before treatment, Fig. 7a, b).

\section{Discussion}

There is much interest in the role of mitochondrial dynamics in cardiac physiology and pathology. However, the upstream
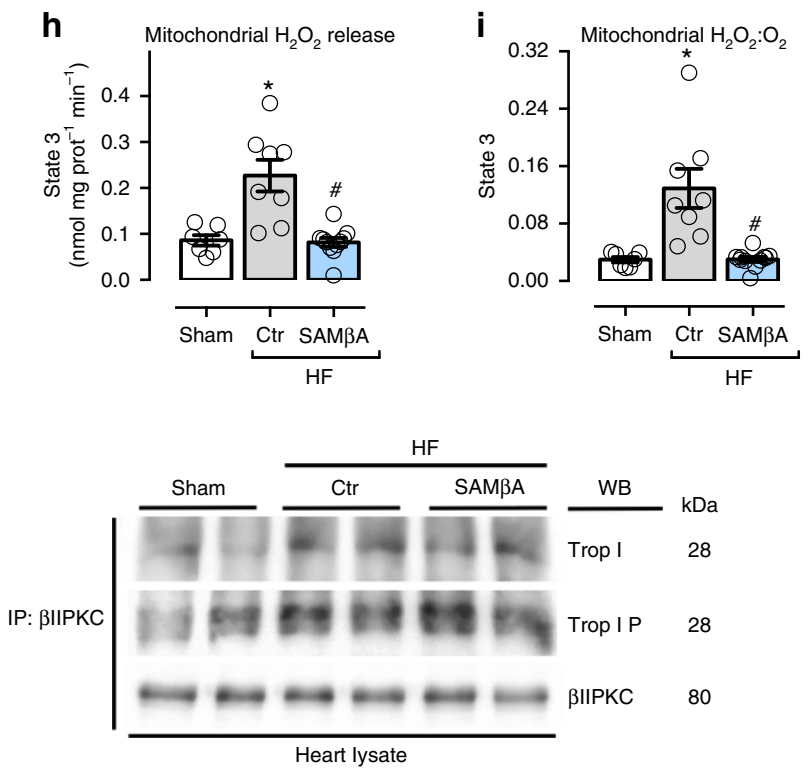

molecular mechanisms affecting mitochondrial morphology in cardiac diseases are largely unknown. The preclinical studies described here suggest that $\beta$ IIPKC activation contributes to heart failure by inhibiting Mfn1, therefore causing accumulation of fragmented/dysfunctional mitochondria, most likely resulting from impaired mitochondrial fusion.

Our previous studies demonstrated that $\beta I I P K C$, a serinethreonine protein kinase $\mathrm{C}$ isozyme, is elevated in human failing hearts ${ }^{11}$ and plays a critical role in the progression of heart failure in rodents $^{12}$. $\beta$ IIPKC activation was also previously demonstrated to be involved in changes in mitochondrial morphology in MEFs upon 
Fig. 6 SAM $\beta A$ treatment reduces mitochondrial fragmentation in heart failure. a Schematic panel of heart failure induction by myocardial infarction (MI) and the treatment protocol. Twelve-week-old male rats were subjected to $\mathrm{MI}$-induced heart failure by left anterior descending coronary artery permanent ligation. Four weeks after $\mathrm{MI}$ induction, the rats were treated with the either SAM $\beta$ A peptide (a selective antagonist of Mfn1- $\beta$ IIPKC association) or with a control peptide (TAT, used to deliver SAM $\beta$ A into the heart). Peptide treatment was continuous for 6 weeks using an Alzet pump delivery at 3 mg per Kg per day. $\mathbf{b}$ Left ventricular ejection fraction and $\mathbf{c}$ LVEDd [left ventricular end-diastolic dimension] measured by echocardiography at the end of the experimental protocol, input: delta of measurements performed before and after treatment; $\mathbf{d}$ representative cardiac transmission electron micrographs (scale bar: $1 \mu \mathrm{m}$ ); e quantification of mitochondrial number and area in the transmission electron micrographs, the analysis was done in a blinded fashion; f mitochondrial respiratory control ratio, $\mathbf{g}$ state 3-dependent oxygen control rate, $\mathbf{h}$ absolute $\mathrm{H}_{2} \mathrm{O}_{2}$ release and $\mathbf{i} \mathrm{H}_{2} \mathrm{O}_{2}: \mathrm{O}_{2}$ in heart samples from sham (white bars, $n=7$ ), TAT-treated heart failure (HF-Ctr, gray bars, $n=8$ ), and SAM $\beta A$-treated heart failure (HF-SAM $\beta A$, blue bars, $n=11)$. $\mathbf{j}$ Cardiac mitochondrial $\beta$ IIPKC immunoprecipitate probed against anti- $\beta$ IIPKC, Mfn1 and phosphorylated serine/threonine antibodies; and $\beta$ IIPKC immunoprecipitate from heart lysate probed with anti- $\beta$ IIPKC, troponin I and phosphorylated troponin I antibodies (representative blot of three independent experiments) from sham, HF-Ctr and HF-SAM $\beta$ A groups. Biochemical measurements were performed in the cardiac remote (viable) zone. These measurements were performed at the end of the experimental protocol. Data are means \pm SEM. ${ }^{\star} P<0.05$ vs. Sham rats. $\# P<0.05$ vs. HF-Ctr rats. One-way or two-way analyses of variance (ANOVA) with post-hoc testing by Duncan. For all the cardiac function studies, the observer was blinded to the experimental groups

a

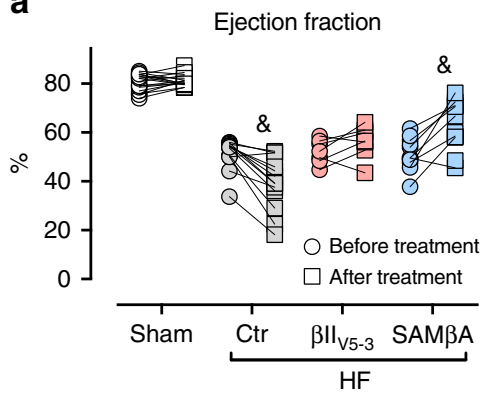

b

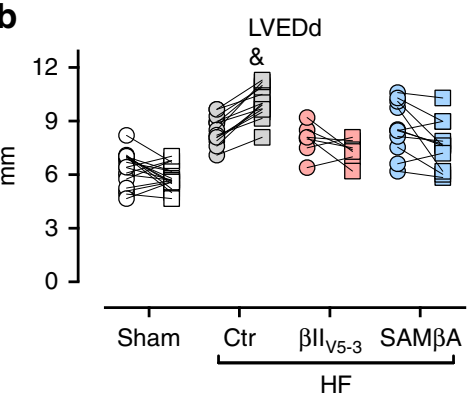

C
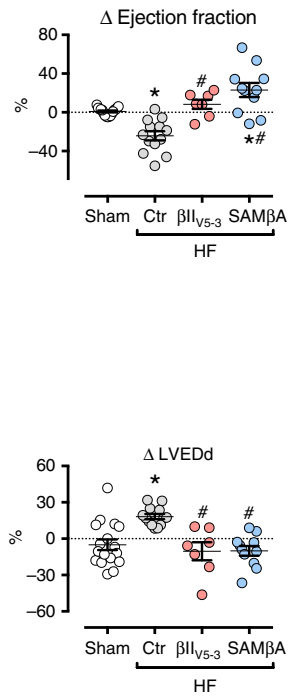

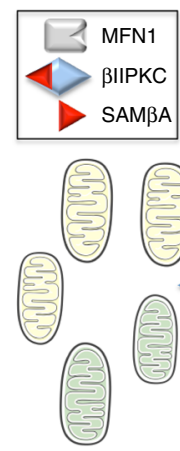

Heart failure

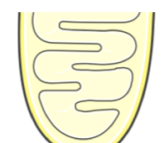

Heart failure + SAM $\beta A$
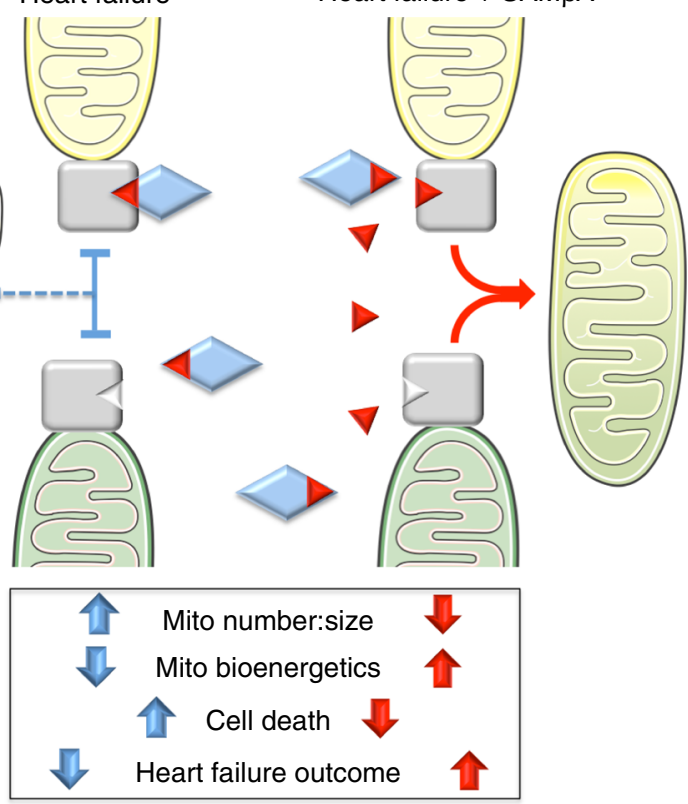

Fig. 7 Sustained SAM $\beta$ A treatment results in better heart failure outcome. a Left ventricular ejection fraction and b LVEDd (left ventricular end-diastolic dimension) measured by echocardiography before and after treatment; input: delta of measurements performed before and after treatment, in animals from sham (white bars, $n=7$ ), TAT-treated heart failure (HF-Ctr, gray bars, $n=9)$, $\beta \|_{V 5-3}$-treated heart failure $\left(\mathrm{HF}-\beta \|_{V 5-3}\right.$, red bars, $\left.n=7\right)$, and SAM $\beta A$ treated heart failure groups (HF-SAM $\beta$ A, blue bars, $n=11$ ). Data are means \pm SEM. ${ }^{\star} P<0.05$ vs. Sham rats. $\# P<0.05$ vs. HF-Ctr rats. $\& P<0.05$ vs. before treatment. One-way or two-way analyses of variance (ANOVA) with post-hoc testing by Duncan. For all the cardiac function studies, the observer was blinded to the experimental groups. c Proposed model for the SAM $\beta$ A-mediated cardioprotection in post-myocardial infarction-induced heart failure

oxidative conditions ${ }^{29}$. Here we identified at least one downstream $\beta$ IIPKC substrate that mediates cardiac pathophysiology. We demonstrated that during heart failure, $\beta$ IIPKC translocated to the mitochondrial outer membrane, where it is bound to and phosphorylated Mfn1. The phosphorylation of Mfn1 by $\beta$ IIPKC at S86 located in the GTPase domain in Mfn1, correlated with a reduction of Mfn1 GTPase activity, but also correlated with an increase in Mfn1 levels at the mitochondria. This is in contrast to Mfn1 phosphorylation by ERK, which also induces mitochondrial fragmentation in $\mathrm{MEFs}^{30}$, but it drives Mfn1-increased clearance thorough ubiquitin proteasome system ${ }^{31,32}$. The accumulation of BIIPKC-phosphorylated Mfn1 could be due to the site of phosphorylation and or because failing hearts have impaired both proteasomal proteolytic activity ${ }^{11}$ and autophagy ${ }^{33}$. Regardless of the mechanism, $\beta$ IIPKC phosphorylation of Mfn1 impairs its GTPase activity, which is critical for mitochondrial fusion activity ${ }^{34}$. Disruption of Mfn1 activity leads to metabolic dysfunction ${ }^{35}$.
Finally, we confirmed that Mfn1 phosphorylation and inhibition by $\beta$ IIPKC alone is a major contributor to the pathophysiology associated with heart failure in this post-myocardial infarction heart failure rat model, using SAM $\beta A$, a rationally designed selective antagonist of Mfn1- IIPKC association. This inhibitory peptide is composed of a 9-amino-acid peptide (RNAENFDRF; $\beta$ IIPKC $_{624-632}$ ) conjugated to the cell permeable peptide $\mathrm{TAT}_{47-57^{36}}$. A 6-week treatment of myocardial infarctioninduced heart failure rats with SAM $\beta A$, starting 4 weeks after myocardial infarction at a time when ventricular remodeling and cardiac dysfunction were present, re-established mitochondrial morphology, bioenergetics, and redox balance. In addition, SAM $\beta A$ had a superior effect on cardiac function, left ventricular compliance, and diastolic function compared to the global $\beta \mathrm{IIPKC}$ inhibitor, $\beta \mathrm{II}_{\mathrm{V} 5-3}$.

Our previous studies showed that preventing accumulation of fragmented mitochondria using other pharmacological means is 
also beneficial. A peptide inhibitor of phosphorylation of Drp1 by $\delta$ PKC, $\delta$ V1-1 or an inhibitor of Drp1 interaction with Fis1, P110, protect neurons and cardiomyocytes from acute ischemic stress $^{37-39}$ and a rationally designed peptide activator of Mfn2 corrects mitochondrial fusion defects in a model of CharcotMarie-Tooth model in neurons ${ }^{40}$. Therefore, the molecular basis of increased mitochondrial fragmentation can be diverse, depending on the disease. However, in all these cases, correcting the mitochondrial morphology improves outcome, by correcting mitochondrial functions and ATP production. We suggest that the improvement in mitochondrial functions is critical for cellular health and that the benefit we saw with sustained treatment using SAM $B A$ on reversal of the cardiac pathological remodeling and dysfunction induced by post-myocardial infarction is due to increased repair ability of the cells. From a clinical standpoint, future experiments checking the effectiveness of SAM $\beta A$ in preventing the development of heart failure by starting the treatment immediately after myocardial infarction are needed. It is also important to validate the cardiac benefits of SAM $\beta$ A by looking at different models of cardiomyopathy and heart failure.

In conclusion, our findings of increased mitochondrial fragmentation in failing hearts and its dependence on BIIPKC activation suggest that $\beta$ IIPKC is a regulator of mitochondrial dynamics through Mfn1 phosphorylation, at least under pathological conditions. Our data also suggest that correcting impaired mitochondrial dynamics, in general, and selectively inhibiting Mfn1- $\beta I I P K C$ interaction with drugs, such as SAM $\beta A$, may provide useful treatments for patients with established heart failure.

\section{Methods}

Sequence alignments. Sequences from different species were aligned using FASTA server ${ }^{41}$, using the following $\beta$ IIPKC proteins: human (P05771-2), mouse (55977813), rat (gi66724), and chicken (A0A1D5PUY5). aPKC proteins: human (P17252), mouse (P20444), rat (P05696), and chicken (Q5F3X1). $\gamma$ PKC proteins: human (P05129), mouse (P63318), and rat (P63319). R3HCC1 proteins: human (Q9Y3T6), mouse (Q8BSI6), rat (Q9EQN5), and chicken (118101322). VAM-1 proteins: human (Q9NZW5), mouse (Q9JLB0), and rat (201066397). MFN1 proteins: human (26251799), mouse (34784646), rat (20376820), and chicken (F1NV08). MFN2 proteins: human (189083768) and $\beta$ IPKC proteins: human (P05771-1).

Animals. The animal protocols were approved by the Stanford University Institutional Animal Care and Use Committee (Protocol ID: 14746) and by the Ethical Committee of Biomedical Sciences Institute of University of São Paulo (2013/134). This study was conducted in accordance with the Guide for the Care and Use of Laboratory Animals published by the US-National Institutes of Health and with the ethical principles in animal research adopted by the Brazilian Society of Laboratory Animal (www.cobea.org.br). The study with $\beta \mathrm{II}_{\mathrm{V} 5-3}$ treatment in male rats included three groups: sham, $n=10$; TAT-treated (control) heart failure, $n=10 ; \beta \mathrm{II}_{\mathrm{V} 5-3^{-}}$ treated heart failure, $n=10$. The study with SAM $\beta$ A treatment included sham, $n=$ 7; TAT-treated (control) heart failure, $n=8$ and SAM $\beta$ A-treated heart failure, $n=$ 11. No animals were excluded from the study. Sham and myocardial infarction surgery were done on the same day, usually 10 rats per day. Four weeks after myocardial infarction, rats were randomized to drug treatments.

Myocardial infarction-induced heart failure. We have chosen this rat model since myocardial infarction is the underlying etiology of heart failure in nearly $70 \%$ of patients ${ }^{42}$. Myocardial infarction was induced by a permanent ligation of the left anterior descending coronary artery in Wistar normotensive male rats at 12 weeks of age ${ }^{43}$. Rats were anesthetized intraperitoneally with ketamine $\left(50 \mathrm{mg} \mathrm{Kg}^{-1}\right)$ and xylazine $\left(10 \mathrm{mg} \mathrm{Kg}^{-1}\right)$, endotracheally intubated, and mechanically ventilated with room air (respiratory rate of 60-70 breaths per minute and tidal volume of $2.5 \mathrm{~mL}$ ). Left thoracotomy between the fourth and fifth ribs was performed and the left anterior descending coronary artery was permanently ligated ${ }^{44}$. Four weeks later, echocardiography (Acuson Sequoia, $14 \mathrm{MHz}$ ) to evaluate fractional shortening was performed and rats showing heart failure symptoms were included in the study. Heart failure was defined when animal presented pathological cardiac remodeling accompanied by left ventricle dysfunction and cardiac dilation, according to the Guidelines of American Heart Association ${ }^{45}$. Left thoracotomy with equal procedure duration, but without left anterior descending coronary artery ligation, was undertaken in the sham group.
Study design. Heart failure animals were treated between the ages of 16 and 22 weeks. Rats were randomized to the treatment groups. Each group was treated with $\mathrm{TAT}_{47-57}-\beta \mathrm{II}_{\mathrm{V} 5-3}$ peptide (global $\beta \mathrm{IIPKC}$ inhibitor, $3 \mathrm{mg}$ per $\mathrm{Kg}$ per day), $\mathrm{TAT}_{47-57}$-SAM $\beta$ A peptide Mfn1- $\beta$ IIPKC inhibitor, $3 \mathrm{mg}$ per Kg per day) or equimolar concentration of $\mathrm{TAT}_{47-57}$-carrier peptide, using Alzet osmotic pumps under the skin on the back of the animal, and were replaced every 2 weeks. The

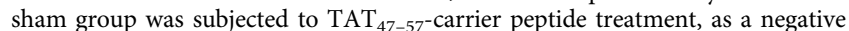
control. At the end of the protocol, cardiac function was re-evaluated by observer blinded to the treatment groups. Forty-eight hours later, all rats were euthanized by decapitation for other analyses. Hearts were dissected into infarct and viable zones using the following criteria. The infarct zone was the left ventricular wall that was thinning and included visible infarct scar, and the viable (remote) zone was the part of the left ventricular wall that was reddish-brown and with normal thickness. All biochemical measurements were performed in the left ventricular viable (remote) zone.

Cardiovascular measurements. Systolic blood pressure was determined noninvasively, using a computerized tail-cuff system (BP-2000, Visitech System). Noninvasive cardiac function evaluation was performed by M-mode echocardiography in anesthetized (isoflurane 3\%) rats. Briefly, rats were positioned in the supine position with front paws wide open and ultrasound transmission gel was applied to the precordium. Transthoracic echocardiography was performed using an Acuson Sequoia model 512 echocardiographer (SIEMENS) equipped with a $14-\mathrm{MHz}$ linear transducer. Left ventricle systolic function was estimated by ejection fraction (EF) and fractional shortening (FS) as follows: $\mathrm{EF}(\%)=\left[\left(\mathrm{LVEDD}^{3}-\mathrm{LVESD}^{3}\right)\right.$ LVEDD $\left.^{3-1)}\right] \times 100$ and FS $(\%)=\left[\left(\right.\right.$ LVEDD-LVESD) LVEDD $\left.^{(-1)}\right] \times 100$, where LVEDD is the left ventricular end-diastolic diameter, and LVESD is the left ventricular end-systolic diameter. The observer was blinded to the treatment groups.

Isolation of adult rat cardiomyocytes. Ten weeks after MI surgery, control, vehicle-treated $\mathrm{HF}$ and $\beta \mathrm{II}_{\mathrm{V} 5-3}$-treated $\mathrm{HF}$ rats were injected with heparin (2000 U $\left.\mathrm{kg}^{-1} \mathrm{IP}\right)$, anesthetized with sodium pentobarbital $\left(100 \mathrm{mg} \mathrm{kg}^{-1} \mathrm{IP}\right)$ and euthanized by decapitation. The hearts were rapidly excised and then perfused with low-Ca ${ }^{2+}$ solution $1\left(100 \mathrm{mM} \mathrm{NaCl}, 10 \mathrm{mM} \mathrm{KCl}, 1.2 \mathrm{mM} \mathrm{KH}{ }_{2} \mathrm{PO}_{4}, 5 \mathrm{mM} \mathrm{MgSO}_{4}, 20 \mathrm{mM}\right.$ glucose, $50 \mathrm{mM}$ taurine, $10 \mathrm{mM}$ HEPES, and $100 \mu \mathrm{M} \mathrm{CaCl}_{2}$ ), then with digestion solution containing low- $\mathrm{Ca}^{2+}$ solution 1 , collagenase $\left(0.5 \mathrm{mg} \mathrm{mL}^{-1}\right.$, Worthington Type $1 \mathrm{~A})$, and protease type XIV $\left(0.04 \mathrm{mg} \mathrm{mL}^{-1}\right.$; Sigma). Following perfusion, the ventricles were cut into fragments $\left(2-5 \mathrm{~mm}^{3}\right)$ in digestion solution. The cell suspension was then filtered through a nylon sieve and centrifuged for $1 \mathrm{~min}$ (at $300-400 \times g$ ) at room temperature. Cell pellets were resuspended in solution 1 containing $125 \mathrm{mg} \mathrm{BSA}$ and $500 \mu \mathrm{M} \mathrm{CaCl}_{2}$.

Cardiomyocyte shortening and relengthening. Cell contraction properties of adult cardiomyocytes were evaluated with a video-based sarcomere spacing acquisition system (SarcLen, IonOptix) ${ }^{46}$. Changes in sarcomere length were recorded and analyzed using IonWizard software (IonOptix). Sarcomeric shortening was determined under basal conditions.

Mitochondrial isolation. Cardiac samples from remote area were minced and homogenized in isotonic mitochondrial buffer $(300 \mathrm{mM}$ sucrose, $10 \mathrm{mM}$ Hepes, 2 mM EGTA, pH $7.2,4^{\circ} \mathrm{C}$ ) containing $0.1 \mathrm{mg} \mathrm{mL}^{-1}$ of type I protease (bovine pancreas, Sigma P4630) to release mitochondria from within muscle fibers and later washed in the same buffer in the presence of $1 \mathrm{mg} \mathrm{mL}^{-1}$ bovine serum albumin ${ }^{47}$. The suspension was homogenized in a $40 \mathrm{~mL}$ tissue grinder and centrifuged at $950 \times g$ for $5 \mathrm{~min}$. The resulting supernatant was centrifuged at $9500 \times g$ for $10 \mathrm{~min}$. The mitochondrial pellet was washed, resuspended in isolation buffer and submitted to a new centrifugation $(9500 \times g$ for $10 \mathrm{~min})$. The mitochondrial pellet was washed and the final pellet was resuspended in a minimal volume of isolation buffer.

Mitochondrial function. All experiments with isolated mitochondria $(0.125 \mathrm{mg}$ mitochondrial protein $\mathrm{mL}^{-1}$ ) were monitored in experimental buffer containing $125 \mathrm{mM}$ sucrose, $65 \mathrm{mM} \mathrm{KCl}, 10 \mathrm{mM}$ Hepes, $2 \mathrm{mM}$ inorganic phosphate, $2 \mathrm{mM}$ $\mathrm{MgCl}_{2}, 100 \mu \mathrm{M}$ EGTA, and $0.01 \% \mathrm{BSA}, \mathrm{pH} 7.2$ and were performed in the presence of succinate, malate, and glutamate substrates ( $2 \mathrm{mM}$ of each) with continuous stirring at $37^{\circ} \mathrm{C}^{48}$. Mitochondrial $\mathrm{O}_{2}$ consumption was monitored using a computer-interfaced Clark-type electrode (OROBOROS Oxygraph-2k). ADP (1 $\mathrm{mM}$-Amresco 0160) was added to induce State 3 respiratory rates. A subsequent addition of oligomycin $(1 \mu \mathrm{g} \mathrm{mL}-1$-Sigma 4876$)$ was used to determine State 4 rates. Respiratory control ratios were calculated by the State 3:State 4 ratio. Additionally, 0.1 mM FCCP [Carbonyl cyanide 4-(trifluoromethoxy) phenylhydrazone-Enzo BML-CM120] was added in order to evaluate $\mathrm{O}_{2}$ consumption in uncoupled mitochondria ${ }^{48}$. Mitochondrial $\mathrm{H}_{2} \mathrm{O}_{2}$ release was measured using Amplex Red ( $25 \mu \mathrm{M}$-Molecular Probes A12222)-horseradish peroxidase $\left(0.5 \mathrm{U} \mathrm{mL}^{-1}\right.$ - Sigma P8125) system ${ }^{48}$. Amplex Red is oxidized in the presence of extramitochondrial horseradish peroxidase bound to $\mathrm{H}_{2} \mathrm{O}_{2}$, generating resorufin, which can be detected using a fluorescence spectrophotometer $\left(\kappa_{\mathrm{ex}}=563 / \kappa_{\mathrm{em}}=\right.$ $587 \mathrm{~nm}$ ) (F-2500 Hitachi-Hitachi). To estimate $\mathrm{H}_{2} \mathrm{O}_{2}$ release during State 3 and State 4 respiratory rates, and in uncoupled mitochondria, we added ADP (1 mM), 
oligomycin $(1 \mu \mathrm{g} \mathrm{mL}-1)$, and FCCP $(0.1 \mathrm{mM})$, respectively. Calibration was conducted by adding $\mathrm{H}_{2} \mathrm{O}_{2}$ at known concentrations $\left(A_{240}=43.6 \mathrm{M}^{-1} . \mathrm{cm}^{-1}\right)$ to the experimental buffer.

Immunoblotting. Cardiac samples were subjected to SDS-PAGE in polyacrylamide gels (6-15\%) depending upon protein molecular weight. After electrophoresis, proteins were electrotransferred to nitrocellulose membranes. Equal gel loading and transfer efficiency were monitored using $0.5 \%$ Ponceau S staining of blot membrane. Blotted membrane was then blocked (5\% BSA, $10 \mathrm{mM}$ Tris-HCl; $\mathrm{pH}$ 7.6), $150 \mathrm{mM} \mathrm{NaCl}$, and $0.1 \%$ Tween 20) for $2 \mathrm{~h}$ at room temperature and then incubated overnight at $4{ }^{\circ} \mathrm{C}$ with specific antibodies against Drp1 (611113, dilution 1:1000) from BD Biosciences; GAPDH (RGM2, dilution 1:10000) from Advanced Immunochemical; Mfn1 (H00055669-M04, dilution 1:1000), Mfn2 (H00009927M01, dilution 1:1000), Opal (H00004976-M01, dilution 1:1000), and IDH2 (H00003418-M01, dilution 1:1000) from Abnova; ATP5A (Ab14748, dilution 1:1000), NDUFA9 (Ab14713, dilution 1:1000), Ubiquinol (Ab110252, dilution 1:1000), and Troponin I (Ab47003, dilution 1:1000) from Abcam; SQSTM1/p62 (5114, dilution 1:1000) and phospho-Troponin I (serine 23/24-4004, dilution 1:1000) from Cell Signaling Technology; Optineurin (10837-1-AP, dilution 1:1000) and NDP52-CALCOCO2 (12229-1-AP, dilution 1:1000) from Proteintech; Fis1 (210-907-R100, dilution 1:1000) from Enzo Life Sciences and aPKC (sc-208, dilution 1:1000), $\beta$ IPKC (sc-209, dilution 1:1000), $\beta$ IIPKC (sc-210, dilution 1:500), $\delta$ PKC (sc-213, dilution 1:500), عPKC (sc-214, dilution 1:500), RACK1 (sc-10775, dilution 1:500), Tom20 (sc-11021, dilution 1:500), VDAC (sc-32063, dilution 1:500), Enolase (sc-15343, dilution 1:500), ALDH2 (sc-100496, dilution 1:500), and Mfn1 (sc-50330, dilution 1:500) from Santa Cruz Biotechnology. Positive and negative controls for the detection of Mfn1 are shown in Supplementary Figure 4b. Binding of the primary antibody was detected with the use of peroxidaseconjugated secondary antibodies (rabbit, mouse or goat for $2 \mathrm{~h}$ at room temperature, dilution 1:10,000) and developed using enhanced chemiluminescence detected by autoradiography. Quantification analysis of blots was performed with the use of Image J software (Image J Corporation based on NIH image). The protein contents were quantified by the Bradford method ${ }^{49}$. Samples were normalized to relative changes in housekeeping proteins [GAPDH (heart lysate), VDAC (mitochondrial fraction) or IDH2 (mitochondrial fraction)] and expressed as the percent of control. Original blot data are available in the Supplementary information (Supplementary Figures $5-8$ ).

Immunoprecipitation. Cardiac mitochondrial fraction $(0.25 \mathrm{mg}$ protein $)$ and lysate $(0.5 \mathrm{mg}$ protein) were incubated with $2 \mu \mathrm{g}$ of the indicated antibodies in $1 \mathrm{~mL}$ immunoprecipitation lysis buffer $(\mathrm{NaCl} 150 \mathrm{mM}$, EDTA $5 \mathrm{mM}$, Tris- $\mathrm{HCl} 10 \mathrm{mM}$, Triton X100 0.1\%, pH 7.4) for $3 \mathrm{~h}$ at $4^{\circ} \mathrm{C}$, followed by incubation with $50 \mu \mathrm{L}$ of protein A/G agarose beads (sc-2003 Santa Cruz Biotechnology) for $1 \mathrm{~h}$ at $4{ }^{\circ} \mathrm{C}$. After low speed centrifugation, $1000 \times g$ for $5 \mathrm{~min}$ at room temperature, pellet was washed 3 times in $1 \mathrm{~mL}$ immunoprecipitation lysis buffer with low speed centrifugation after each washed. The immunoprecipitates were either assayed for GTPase activity or separated on SDS-PAGE and transferred onto nitrocellulose membranes. The membranes were then probed with the indicated antibodies.

GTPase activity assay. Recombinant Drp1 (H00010059-P01), Mfn1 (H00055669$\mathrm{P} 01$ ), or Mfn2 (H00009927-Q01) from Abnova were incubated with $50 \mathrm{ng}$ of recombinant $\beta$ IIPKC (ab60841) from Abcam in assay buffer $(25 \mathrm{mM}$ Tris- $\mathrm{HCl}, \mathrm{pH}$ 7.5, $1 \mathrm{mM} \mathrm{CaCl} 2,20 \mathrm{mM} \mathrm{MgCl} 2,1 \mathrm{mM}$ DTT, $25 \mathrm{nM} \mathrm{ATP}$ ) in the presence and absence of phosphatidylserine and diacylglycerol PS/DAG for $30 \mathrm{~min}$ at $37^{\circ} \mathrm{C}$. GTPase activity of the proteins was determined using a GTPase assay kit (602-0120, Novus Biologicals) according to manufacturer's instructions. To determine GTPase activity of Mfn1 and Mfn2 in the tissue lysate, a total of $0.25 \mathrm{mg}$ cardiac mitochondria were immunoprecipitated overnight with $2 \mu \mathrm{g}$ of antibodies against Mfn1 or Mfn2 and $50 \mu \mathrm{L}$ of Protein A/G-agarose ${ }^{33}$. After three washes with lysis buffer and three washes with GTPase buffer (50 mM Tris [pH 7.5], $2.5 \mathrm{mM}$ $\mathrm{MgCl}_{2}$, and $0.02 \% 2$ - mercaptoethanol), the beads were incubated with $0.5 \mathrm{mM}$ GTP at $30^{\circ} \mathrm{C}$ for $1 \mathrm{~h}$. The released free phosphate was quantified using the GTPase assay kit, as above.

\section{Phosphorylation assay. Twenty-five nanogram of recombinant Mfn1} (H00055669-P01, Abnova) was incubated with $50 \mathrm{ng}$ of recombinant $\beta$ IPKC (ab60840, Abcam) or BIIPKC (ab60841, Abcam) in assay buffer (25 mM Tris- $\mathrm{HCl}$, $\mathrm{pH} 7.5,1 \mathrm{mM} \mathrm{CaCl}_{2}, 20 \mathrm{mM} \mathrm{MgCl}_{2}, 1 \mathrm{mM} \mathrm{DTT}$, and $25 \mathrm{nM} \mathrm{ATP}$ ) in the presence and absence of phosphatidylserine and diacylglycerol PS/DAG for $30 \mathrm{~min}$ at $37^{\circ} \mathrm{C}$. Mfn1 phosphorylation was evaluated using radioactive $\left[\mathrm{P}^{32}\right] \mathrm{P}^{32}$-ATP incorporation ${ }^{11}$.

Mass spectrometry analysis. The identification of Mfn1 phosphorylation at $\beta$ IIPKC site was performed by incubating recombinant Mfn1 with recombinant $\beta$ IIPKC plus PS/DG/Ca (PKC activators). Phosphopeptide spectra of Mfn1 was examined using LC/MS/MS. LTQ Orbitrap Velos mass spectrometer was used, the nano HPLC was an Eksigent nano 2D with a flow rate of $0.6 \mu \mathrm{L} \mathrm{min}^{-1}$. In solution digestion was done with trypsin overnight. Peptides were further purified on a stagetip using C18 matrix. Data-dependent acquisition was used and the top 10 most intense peptides identified in the survey scan were selected for fragmentation using higher energy collisional-induced dissociation (HCD).

Proteinase K digestion. For proteinase $\mathrm{K}$ digestion, isolated mitochondria were suspended in isotonic mitochondrial buffer and incubated for $15 \mathrm{~min}$ with $5 \mu \mathrm{g} \mathrm{mL} L^{-1}$ proteinase $\mathrm{K}$ at $37^{\circ} \mathrm{C}$. Digestion was terminated by adding $2 \mathrm{mM}$ phenylmethylsulfonyl fluoride. Mitochondrial proteins were separated on SDS-PAGE and transferred onto nitrocellulose membranes. The membranes were then probed with the indicated antibodies.

Transmission electron microscopy. Cardiac samples and culture neonatal rat cardiomyocytes were fixed, embedded, and stained ${ }^{50}$. Small blocks ( 5 blocks per animal) from a cardiac remote area and from neonatal cardiomyocyte pellets were fixed with $2 \%$ glutaraldehyde and $4 \%$ paraformaldehyde in sodium cacodylate buffer, $\mathrm{pH} 7.3$ for $1 \mathrm{~h}$ at room temperature and cut into $\sim 1 \mathrm{~mm}^{3}$ blocks. After several buffer washes, samples were post-fixed in $2 \%$ osmium tetroxide and $1 \%$ uranyl acetate for $2 \mathrm{~h}$, rinsed in water, dehydrated through ascending concentrations of ethanol followed by $100 \%$ acetone, and then infiltrated and embedded in Eponate 12. Final blocks were used to evaluate intermyofibrillar mitochondrial number and size. Images were acquired using a JEOL1230 Gatan 967 CCD transmission electron microscope at $80 \mathrm{kV}$ and a Gatan Orius $4 \mathrm{k} \times 4 \mathrm{k}$ digital camera (Gatan). We performed quantitative analysis in at least 10 fields per rat (3 rats per group) and at least 15 cardiomyocytes per group (3 independent experiments) using Image J software. The analysis was done in a blinded fashion.

Cell culture. WT (ATCC CRL-2991) and Mfn1 knockout (ATCC CRL-2992) MEFs were acquired from ATCC and cultured in Dulbecco's Modification of Eagle's Medium/Ham's F-12 50/50 Mix supplemented with $10 \%$ FBS, $100 \mathrm{U} \mathrm{mL}^{-1}$ penicillin, and $100 \mu \mathrm{g} \mathrm{mL}^{-1}$ streptomycin. Site-directed mutagenesis was performed to generate Mfn1 S86A, S284A and S290A variants using a QuickChange site-directed mutagenesis kit (Stratagene). Mutants were sequenced to ensure that no additional mutations were introduced and then transfected into Mfn1 knockout MEFs using Lipofectamine transfection reagent (Thermo). Primary cultures of neonatal cardiac myocytes were prepared from the heart of 1-day-old rats by gentle digestion at $37^{\circ} \mathrm{C}$ using a cell isolation kit protocol (ac-7018, Cellutron) $)^{39}$. Cells were cultured in the presence of $0.1 \mathrm{mmol} \mathrm{L}^{-1}$ bromodeoxyuridine on primary tissue culture dishes (BD Falcon) or on laminin-coated slides in Dulbeco's modified Eagle's medium with $10 \%$ fetal bovine serum for 4 days. Primary cultures of adult cardiac myocytes were prepared from the heart of 2-month-old rats by enzymatic digestion with collagenase Type II (Worthington) using a Langendorff system ${ }^{51}$. Cells were cultured in M199 medium supplemented with $2 \mathrm{mg} \mathrm{ml}^{-1} \mathrm{BSA}, 1 \mu \mathrm{M}$ insulin, $2 \mathrm{mM}$ carnitine hydrochloride, $5 \mathrm{mM}$ creatine, and $5 \mathrm{mM}$ taurine for $2 \mathrm{~h}$ Small interfering siRNA duplexes for $\beta$ IIPKC or negative control were obtained from Amcon Biotechnology. Cardiac myocytes at $50 \%$ confluency were transfected for $48 \mathrm{~h}$ with siRNA of $\beta$ IIPKC or negative control siRNA using Lipofectamine 2000 (Invitrogen), according to the manufacturer's instructions.

Immunocytochemistry. Cells cultured on coverslips were washed with cold PBS, fixed in $4 \%$ formaldehyde and permeabilized with $0.1 \%$ Triton X-100. After incubation with $2 \%$ normal goat serum (to block non-specific staining), fixed cells were incubated overnight at $4{ }^{\circ} \mathrm{C}$ with antibodies against Tom20 (sc-11021, Santa Cruz Biotechnology, dilution 1:500). Cells were washed with PBS and incubated for 60 min with FITC-labeled goat anti-rabbit antibody (65-6111, Invitrogen, dilution 1:500) followed by incubation with Hoechst dye (1:10000) for 10 min. Coverslips were mounted and slides were imaged using a DeltaVision OMX SR wide field deconvolution fluorescence microscope (Applied Precision Inc). Quantification was carried out using Image J software.

Measurement of cell viability. Cell viability was measured using an in vitro toxicology assay, MTT-based kit (Sigma), according to the manufacturer's instructions. Cell death was detected by measuring lactate dehydrogenase (LDH) activity in the cell culture media. For each assay, the media was combined with Sodium pyruvate $(30 \mathrm{mM}), \mathrm{NADH}(6.6 \mathrm{mM})$, and Tris-HCL $(0.2 \mathrm{M}, \mathrm{pH} 7.3)$, in a final volume to $200 \mu \mathrm{l}$. $\mathrm{LDH}$ activity was determined by a decrease in absorbance at $340 \mathrm{~nm}$ for $10 \mathrm{~min}$ at $25^{\circ} \mathrm{C}$, resulting from the oxidation of NADH.

Peptide synthesis. Peptides were synthesized using solid-phase chemistry on a Liberty Microwave Peptide Synthesizer (CEM Corporation, Matthews) ${ }^{7}$. Peptides were synthesized as one polypeptide with $\mathrm{TAT}_{47-57}$ carrier in the following order: $\mathrm{N}$-terminus-TAT-spacer (Gly-Gly)-cargo-C-terminus.

Statistical analysis. Data are presented as means \pm standard error of the mean (SEM). Data normality was assessed through Shapiro-Wilk test. One-way analysis of variance (ANOVA) was used to analyze data presented in Figs. 1-6. Two-way ANOVA for repeated measures was used to analyze data depicted in Fig. 7. Whenever significant $F$-values were obtained, Duncan adjustment was used for multiple comparison purposes. GraphPad Prism Statistics was used for the analysis and statistical significance was considered achieved when the value of $P$ was $<0.05$. 
Reporting summary. Further information on experimental design is available in the Nature Research Reporting Summary linked to this article.

\section{Data availability}

The data that support the findings of this study are available from the corresponding author upon reasonable request.

Received: 27 November 2017 Accepted: 18 December 2018

Published online: 18 January 2019

\section{References}

1. Gerczuk, P. Z. \& Kloner, R. A. An update on cardioprotection: a review of the latest adjunctive therapies to limit myocardial infarction size in clinical trials. J. Am. Coll. Cardiol. 59, 969-978 (2012).

2. Roger, V. L. et al. Heart disease and stroke statistics-2012 update: a report from the American Heart Association. Circulation 125, e2-e220 (2012).

3. Vlahos, C. J., McDowell, S. A. \& Clerk, A. Kinases as therapeutic targets for heart failure. Nat. Rev. 2, 99-113 (2003).

4. Force, T. \& Kolaja, K. L. Cardiotoxicity of kinase inhibitors: the prediction and translation of preclinical models to clinical outcomes. Nat. Rev. 10, 111-126 (2011).

5. Mochly-Rosen, D., Das, K. \& Grimes, K. V. Protein kinase C, an elusive therapeutic target? Nat. Rev. 11, 937-957 (2012).

6. Qvit, N., Kornfeld, O. S. \& Mochly-Rosen, D. Engineered substrate-specific delta PKC antagonists to enhance cardiac therapeutics. Angew. Chem. Int. Ed. Engl. 55, 15672-15679 (2016).

7. Qvit, N., Disatnik, M. H., Sho, E. \& Mochly-Rosen, D. Selective phosphorylation inhibitor of delta protein kinase C-pyruvate dehydrogenase kinase protein-protein interactions: application for myocardial injury in vivo. J. Am. Chem. Soc. 138, 7626-7635 (2016).

8. Palaniyandi, S. S., Sun, L., Ferreira, J. C. \& Mochly-Rosen, D. Protein kinase C in heart failure: a therapeutic target? Cardiovasc. Res. 82, 229-239 (2009).

9. Bates, E. et al. Intracoronary KAI-9803 as an adjunct to primary percutaneous coronary intervention for acute ST-segment elevation myocardial infarction. Circulation 117, 886-896 (2008).

10. Inagaki, $K$. et al. Inhibition of delta-protein kinase $C$ protects against reperfusion injury of the ischemic heart in vivo. Circulation 108, 2304-2307 (2003).

11. Ferreira, J. C., Boer, B. N., Grinberg, M., Brum, P. C. \& Mochly-Rosen, D. Protein quality control disruption by PKCbetaII in heart failure; rescue by the selective PKCbetaII inhibitor, betaIIV5-3. PLoS One 7, e33175 (2012).

12. Ferreira, J. C. et al. Pharmacological inhibition of betaIIPKC is cardioprotective in late-stage hypertrophy. J. Mol. Cell. Cardiol. 51, 980-987 (2011).

13. Palaniyandi, S. S., Ferreira, J. C. B., Brum, P. C. \& Mochly-Rosen, D. PKCbetaII inhibition attenuates myocardial infarction induced heart failure and is associated with a reduction of fibrosis and pro-inflammatory responses. J. Cell. Mol. Med. 15, 1769-1777 (2011).

14. Ferreira, J. C., Brum, P. C. \& Mochly-Rosen, D. BetaIIPKC and epsilonPKC isozymes as potential pharmacological targets in cardiac hypertrophy and heart failure. J. Mol. Cell. Cardiol. 51, 479-484 (2011).

15. Stebbins, E. G. \& Mochly-Rosen, D. Binding specificity for RACK1 resides in the V5 region of beta II protein kinase C. J. Biol. Chem. 276, 29644-29650 (2001).

16. Ron, D. et al. Cloning of an intracellular receptor for protein kinase C: a homolog of the beta subunit of G proteins. Proc. Natl Acad. Sci. USA 91, 839-843 (1994).

17. Ron, D. \& Mochly-Rosen, D. An autoregulatory region in protein kinase C the pseudoanchoring site. Proc. Natl Acad. Sci. USA 92, 492-496 (1995).

18. Serneri, G. G. et al. Cardiac angiotensin II formation in the clinical course of heart failure and its relationship with left ventricular function. Circ. Res. 88, 961-968 (2001).

19. Peterson L. R., et al. Ceramide remodeling and risk of cardiovascular events and mortality. J Am Heart Assoc 7, e007931 (2018).

20. Parra, V. et al. Changes in mitochondrial dynamics during ceramide-induced cardiomyocyte early apoptosis. Cardiovasc. Res. 77, 387-397 (2008).

21. Qi, J. et al. Mitochondrial fission is required for angiotensin II-induced cardiomyocyte apoptosis mediated by a Sirt1-p53 signaling pathway. Front. Pharmacol. 9, 176 (2018).

22. Wu, S., Zhou, F., Zhang, Z. \& Xing, D. Mitochondrial oxidative stress causes mitochondrial fragmentation via differential modulation of mitochondrial fission-fusion proteins. FEBS J. 278, 941-954 (2011).

23. Qvit, N. \& Mochly-Rosen, D. Highly specific modulators of protein kinase C localization: applications to heart failure. Drug Discov. Today Dis. Mech. 7, e87-e93 (2010)
24. Souroujon, M. C. \& Mochly-Rosen, D. Peptide modulators of proteinprotein interactions in intracellular signaling. Nat. Biotechnol. 16, 919-924 (1998).

25. Qvit, N., Joshi, A. U., Cunningham, A. D., Ferreira, J. C. \& Mochly-Rosen, D. Glyceraldehyde-3-phosphate dehydrogenase (GAPDH) protein-protein interaction inhibitor reveals a non-catalytic role for GAPDH oligomerization in cell death. J. Biol. Chem. 291, 13608-13621 (2016).

26. Huang, X. Treatment of urinary retention with acupuncture and moxibustion. J. Tradit. Chin. Med. 11, 187-188 (1991).

27. Schwarze, S. R., Ho, A., Vocero-Akbani, A. \& Dowdy, S. F. In vivo protein transduction: delivery of a biologically active protein into the mouse. Science 285, 1569-1572 (1999).

28. Lu, Q. W., Hinken, A. C., Patrick, S. E., Solaro, R. J. \& Kobayashi, T. Phosphorylation of cardiac troponin I at protein kinase C site threonine 144 depresses cooperative activation of thin filaments. J. Biol. Chem. 285, 11810-11817 (2010)

29. Pinton, $\mathrm{P}$. et al. Protein kinase $\mathrm{C}$ beta and prolyl isomerase 1 regulate mitochondrial effects of the life-span determinant p66Shc. Science 315, 659-663 (2007)

30. Pyakurel, A., Savoia, C., Hess, D. \& Scorrano, L. Extracellular regulated kinase phosphorylates mitofusin 1 to control mitochondrial morphology and apoptosis. Mol. Cell 58, 244-254 (2015).

31. Leboucher, G. P. et al. Stress-induced phosphorylation and proteasomal degradation of mitofusin 2 facilitates mitochondrial fragmentation and apoptosis. Mol. Cell 47, 547-557 (2012).

32. Tanaka, A. et al. Proteasome and p97 mediate mitophagy and degradation of mitofusins induced by Parkin. J. Cell Biol. 191, 1367-1380 (2010).

33. Campos, J. C. et al. Exercise reestablishes autophagic flux and mitochondrial quality control in heart failure. Autophagy 13, 1304-1317 (2017).

34. Ishihara, N., Eura, Y. \& Mihara, K. Mitofusin 1 and 2 play distinct roles in mitochondrial fusion reactions via GTPase activity. J. Cell Sci. 117, 6535-6546 (2004).

35. Chen, Y., Liu, Y. \& Dorn, G. W. 2nd Mitochondrial fusion is essential for organelle function and cardiac homeostasis. Circ. Res. 109, 1327-1331 (2011).

36. Chen, L. et al. Molecular transporters for peptides: delivery of a cardioprotective epsilonPKC agonist peptide into cells and intact ischemic heart using a transport system, R(7). Chem. Biol. 8, 1123-1129 (2001).

37. Qi, X., Disatnik, M. H., Shen, N., Sobel, R. A. \& Mochly-Rosen, D. Aberrant mitochondrial fission in neurons induced by protein kinase $\mathrm{C}\{$ delta $\}$ under oxidative stress conditions in vivo. Mol. Biol. Cell 22, 256-265 (2011).

38. Qi, X., Qvit, N., Su, Y. C. \& Mochly-Rosen, D. A novel Drp1 inhibitor diminishes aberrant mitochondrial fission and neurotoxicity. J. Cell Sci. 126, 789-802 (2013)

39. Disatnik, M. H. et al. Acute inhibition of excessive mitochondrial fission after myocardial infarction prevents long-term cardiac dysfunction. J. Am. Heart Assoc. 2, e000461 (2013).

40. Franco, A. et al. Correcting mitochondrial fusion by manipulating mitofusin conformations. Nature 540, 74-79 (2016).

41. Pearson, W. R. \& Lipman, D. J. Improved tools for biological sequence comparison. Proc. Natl Acad. Sci. USA 85, 2444-2448 (1988).

42. Gheorghiade, M. \& Bonow, R. O. Chronic heart failure in the United States: a manifestation of coronary artery disease. Circulation 97, 282-289 (1998).

43. Campos, J. C. et al. Exercise training restores cardiac protein quality control in heart failure. PLoS One 7, e52764 (2012).

44. Johns, T. N. \& Olson, B. J. Experimental myocardial infarction. I. A method of coronary occlusion in small animals. Ann. Surg. 140, 675-682 (1954).

45. Hunt, S. A. et al. 2009 focused update incorporated into the ACC/AHA 2005 Guidelines for the Diagnosis and Management of Heart Failure in Adults: a report of the American College of Cardiology Foundation/American Heart Association Task Force on Practice Guidelines: developed in collaboration with the International Society for Heart and Lung Transplantation. Circulation 119, e391-e479 (2009).

46. Gomes, K. M. et al. Aldehyde dehydrogenase 2 activation in heart failure restores mitochondrial function and improves ventricular function and remodelling. Cardiovasc. Res. 103, 498-508 (2014)

47. Cancherini, D. V., Queliconi, B. B. \& Kowaltowski, A. J. Pharmacological and physiological stimuli do not promote $\mathrm{Ca}(2+)$-sensitive $\mathrm{K}+$ channel activity in isolated heart mitochondria. Cardiovasc. Res. 73, 720-728 (2007).

48. Tahara, E. B., Navarete, F. D. \& Kowaltowski, A. J. Tissue-, substrate-, and site-specific characteristics of mitochondrial reactive oxygen species generation. Free Radic. Biol. Med. 46, 1283-1297 (2009).

49. Hammond, J. B. \& Kruger, N. J. The bradford method for protein quantitation. Methods Mol. Biol. 3, 25-32 (1988).

50. Karnovsky, M. J. The localization of cholinesterase activity in rat cardiac muscle by electron microscopy. J. Cell Biol. 23, 217-232 (1964).

51. Bozi L. H. M., et al. Endoplasmic reticulum stress impairs cardiomyocyte contractility through JNK-dependent upregulation of BNIP3. Int. J. Cardiol. 272, 194-201 (2018). 


\section{Acknowledgements}

This work was supported by the National Institute of Health Grant HL52141 to D.M.-R.; Fundação de Amparo à Pesquisa do Estado de São Paulo-Brasil (FAPESP \#2009/123492, \#2010/00028-4, \#2010/51906-1, \#2012/05765-2, \#2015/20783-5, \#2016/01633-5, \#2015/22814-5, \#2016/09611-0, \#2017/16694-2, \#2017/11142-1, and \#2017/16540-5 to J. C.B.F.), Conselho Nacional de Pesquisa e Desenvolvimento (CNPq) - Brasil [\#303281/ 2015-4, \#470880/2012-0, and \#407306/2013-7 to J.C.B.F.], Coordenação de Aperfeiçoamento de Pessoal de Nível Superior-Brasil (CAPES)_Finance Code 001; and Instituto Nacional de Ciência e Tecnologia and Centro de Pesquisa e Desenvolvimento de Processos Redox em Biomedicina. We thank Camille C. Caldeira-da-Silva for technical assistance.

\section{Author contributions}

J.C.B.F and D.M.R. designed the study, designed the inhibitor, and wrote the manuscript. J.C.B.F performed functional studies of peptides $\beta \mathrm{II}_{\mathrm{V} 5-3}$ and $\mathrm{SAM} \beta \mathrm{A}$ in vivo and in culture. N.Q. designed SAM $\beta$ A peptide and generated structural images. X.Q. performed Co-IP studies and in vitro GTPase assays. J.C.C., V.M.L, B.B.Q., L.H.M.B., and A.J.K performed animal surgeries and structural and functional studies of mitochondria in heart failure. L.R.G.B. performed point mutation studies in MEFs. M.H.D. performed in vitro Co-IP and phosphorylation assays. P.M.M.D. performed echocardiogram measurements.

\section{Additional information}

Supplementary Information accompanies this paper at https://doi.org/10.1038/s41467018-08276-6

Competing interests: The authors declare no competing interests.

Reprints and permission information is available online at http://npg.nature.com/ reprintsandpermissions/

Journal peer review information: Nature Communications thanks the anonymous reviewers for their contributions to the peer review of this work. Peer reviewer reports are available.

Publisher's note: Springer Nature remains neutral with regard to jurisdictional claims in published maps and institutional affiliations.

(c) (i) adaptation, distribution and reproduction in any medium or format, as long as you give appropriate credit to the original author(s) and the source, provide a link to the Creative Commons license, and indicate if changes were made. The images or other third party material in this article are included in the article's Creative Commons license, unless indicated otherwise in a credit line to the material. If material is not included in the article's Creative Commons license and your intended use is not permitted by statutory regulation or exceeds the permitted use, you will need to obtain permission directly from the copyright holder. To view a copy of this license, visit http://creativecommons.org/ licenses/by/4.0/.

(C) The Author(s) 2019 